LLNL ARRA Report on CCSI Uncertainty Quantification Capabilities with Applications on MEA Process Models

B. M. Ng, C. H. Tong, J. C. Ou, J. R. Leek

February 27, 2012 
This document was prepared as an account of work sponsored by an agency of the United States government. Neither the United States government nor Lawrence Livermore National Security, LLC, nor any of their employees makes any warranty, expressed or implied, or assumes any legal liability or responsibility for the accuracy, completeness, or usefulness of any information, apparatus, product, or process disclosed, or represents that its use would not infringe privately owned rights. Reference herein to any specific commercial product, process, or service by trade name, trademark, manufacturer, or otherwise does not necessarily constitute or imply its endorsement, recommendation, or favoring by the United States government or Lawrence Livermore National Security, LLC. The views and opinions of authors expressed herein do not necessarily state or reflect those of the United States government or Lawrence Livermore National Security, LLC, and shall not be used for advertising or product endorsement purposes.

This work performed under the auspices of the U.S. Department of Energy by Lawrence Livermore National Laboratory under Contract DE-AC52-07NA27344. 

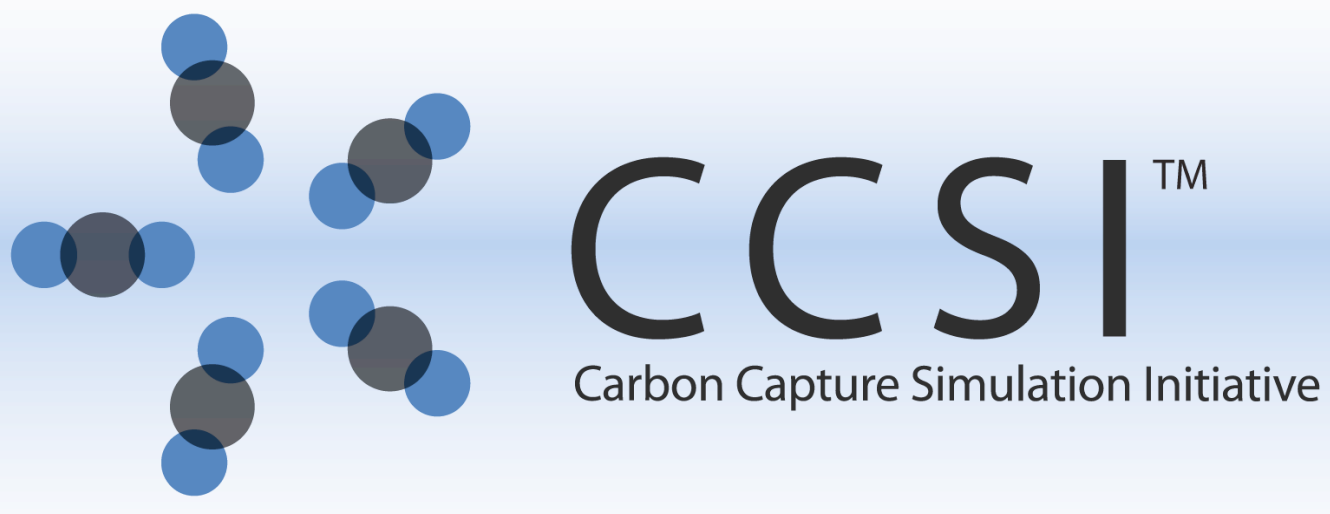

\title{
LLNL ARRA Report on CCSI Uncertainty Quantification Capabilities with Applications on MEA Process Models
}

\author{
Work Performed Under \\ Activity Number 0004000.6.600.007.002 ARRA \\ Prepared by \\ Brenda Ng, Charles Tong, Jeremy Ou, Jim Leek \\ Lawrence Livermore National Laboratory \\ Livermore, CA 94550
}

In collaboration with the National Energy Technology Laboratory, Los Alamos National Laboratory, and Pacific Northwest National Laboratory

Prepared for

U.S. Department of Energy

National Energy Technology Laboratory

24 February 2012
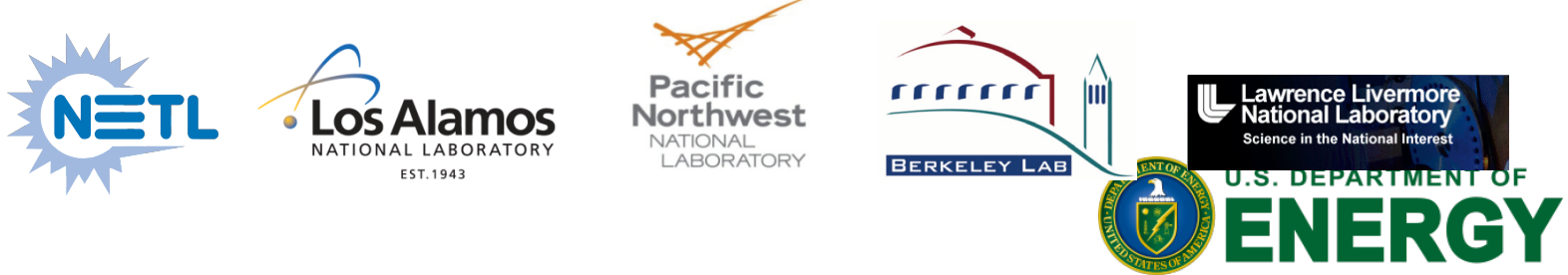


\section{Revision Log}

\begin{tabular}{|c|c|c|c|}
\hline Revision & Date & Revised By: & Description \\
\hline 0 & $2 / 24 / 2012$ & Brenda Ng & Original \\
\hline
\end{tabular}

\section{Disclaimer}

This report was prepared as an account of work sponsored by an agency of the United States Government. Neither the United States Government nor any agency thereof, nor any of their employees, makes any warranty, express or implied, or assumes any legal liability or responsibility for the accuracy, completeness, or usefulness of any information, apparatus, product, or process disclosed, or represents that its use would not infringe privately owned rights. Reference herein to any specific commercial product, process, or service by trade name, trademark, manufacturer, or otherwise does not necessarily constitute or imply its endorsement, recommendation, or favoring by the United States Government or any agency thereof. The views and opinions of authors expressed herein do not necessarily state or reflect those of the United States Government or any agency thereof.

\section{Acknowledgement of Funding}

This project was funded under the Carbon Capture Simulation Initiative under the following FWP's and contracts:

LANL - FE-101-002-FY10

PNNL - 60115

LLNL - FEW0180

LBNL - CSNW1130

NETL - RES-0004000.6.600.007.002 


\section{Table of Contents}

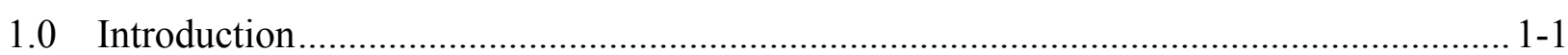

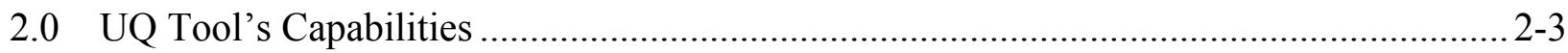

3.0 UQ Graphical User Interface ……...................................................................... 3-6

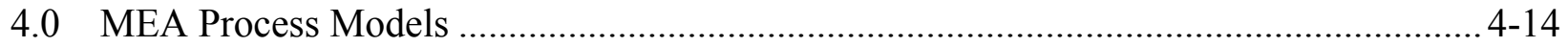

5.0 UQ Analyses on MEA Process Data ……………….............................................. 5-22

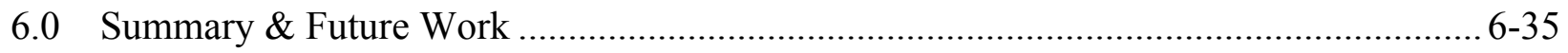

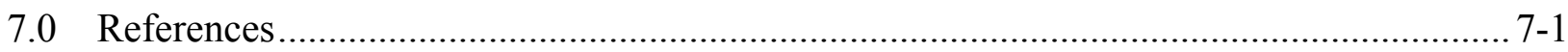

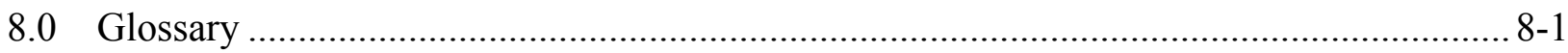

\section{List of Figures}

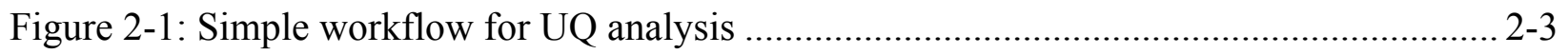

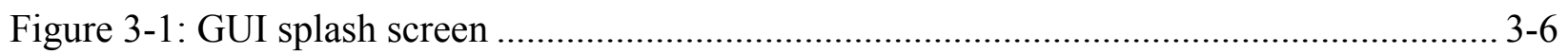

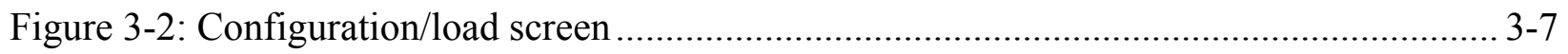

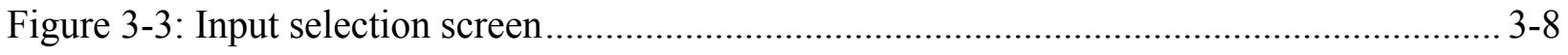

Figure 3-4: Output selection screen ............................................................................. 3-8

Figure 3-5: Sampling scheme selection screen ................................................................... 3-9

Figure 3-6: Sample preview screen.............................................................................. 3-10

Figure 3-7: Sample generation progress and UQ method selection screen ............................... 3-11

Figure 3-8: Uncertainty analysis results screen ................................................................ 3-11

Figure 3-9: Scatter plot screen ...................................................................................... 3-12

Figure 3-10: Variance-based sensitivity analysis screen ...................................................... 3-12

Figure 3-11: Response surface validation screen............................................................... 3-13

Figure 3-12: Response surface visualization screen ........................................................... 3-13

Figure 4-1: Schematic of a packed column ......................................................................... 4-15

Figure 4-2: Experimental data from (Billet \& Schultes, 1993) .............................................. 4-17 
Figure 4-3: Experimental data from (Hanley and Chen, 2012) ........................................ 4-20

Figure 5-1: Output PDF and CDF from forward uncertainty analysis for Model V1, Case 1... 5-23

Figure 5-2: Validation plots from response surface analysis for Model V1, Case 1 ............... 5-24

Figure 5-3: 3-D response surface visualization for Model V1, Case 1................................. 5-24

Figure 5-4: Output PDF from forward uncertainty analysis for Model V1, Case 2 ................ 5-25

Figure 5-5: Pie chart from sensitivity analysis for Model V1, Case 2............................... 5-25

Figure 5-6: Scatter plots from sensitivity analysis for Model V1, Case 2 ........................... 5-26

Figure 5-7: Output PDF from forward uncertainty analysis for Model V1, Case 3 ................ 5-26

Figure 5-8: Bootstrap modified means plot from parameter screening for Model V1, Case 3 . 5-27

Figure 5-9: Input posteriors from Bayesian inference for Model V1, Case 3 ........................ 5-27

Figure 5-10: Output PDF and CDF from forward uncertainty analysis for Model V2, Case 1. 5-29

Figure 5-11: Validation plots from response surface analysis for Model V2, Case 1 ............ 5-29

Figure 5-12: Scatter plots from sensitivity analysis analysis for Model V2, Case 1 .............. 5-30

Figure 5-13: 2-D response surface visualization for Model V2, Case 1............................... 5-30

Figure 5-14: Output PDF and CDF from forward uncertainty analysis for Model V2, Case 2. 5-31

Figure 5-15: Validation plots from response surface analysis for Model V2, Case 2 ............. 5-31

Figure 5-16: Scatter plots from sensitivity analysis analysis for Model V2, Case 2 ............... 5-32

Figure 5-17: 2-D response surface visualization for Model V2, Case 2.............................. 5-32

Figure 5-18: Output PDF from forward uncertainty analysis for Model V2, Case 3 .............. 5-33

Figure 5-19: Validation plots from response surface analysis for Model V2, Case 3 ............. 5-33

Figure 5-20: Scatter plots from sensitivity analysis analysis for Model V2, Case 3 ............... 5-34

Figure 5-21: 1-D response surface visualization for Model V2, Case 3............................. 5-34 


\section{List of Tables}

Table 4-1: Equilibrium reactions from Aspen MEA insert ................................................. 4-14

Table 4-2: Equilibrium constant parameters from Aspen MEA insert ............................... 4-15

Table 4-3: Terms in the Billet and Schultes correlation ................................................... 4-16

Table 4-4: Kinetic reactions from Aspen MEA-REA insert.............................................. 4-18

Table 4-5: Equilibrium reactions from Aspen MEA-REA insert ........................................ 4-19

Table 4-6: Terms in the Hanley correlation ....................................................................... 4-20

Table 5-1: Input parameters for Model V1 ................................................................. 5-22

Table 5-2: Sample size of Model V1 simulation data................................................... 5-23

Table 5-3: Input parameters for Model V2 ............................................................ 5-28

Table 5-4: Sample size of Model V2 simulation data...................................................... 5-28 


\subsection{Introduction}

\subsection{BACKGROUND}

The Carbon Capture Simulation Initiative (CCSI) is a partnership among national laboratories, industry and academic institutions that will develop and deploy state-of-the-art computational modeling and simulation tools to accelerate the commercialization of carbon capture technologies from discovery to development, demonstration, and ultimately the widespread deployment to hundreds of power plants. The CCSI Toolset will provide end users in industry with a comprehensive, integrated suite of scientifically validated models with uncertainty quantification, optimization, risk analysis and decision making capabilities. The CCSI Toolset will incorporate commercial and open-source software currently in use by industry and will also develop new software tools as necessary to fill technology gaps identified during execution of the project. The CCSI Toolset will (1) enable promising concepts to be more quickly identified through rapid computational screening of devices and processes; (2) reduce the time to design and troubleshoot new devices and processes; (3) quantify the technical risk in taking technology from laboratory-scale to commercial-scale; and (4) stabilize deployment costs more quickly by replacing some of the physical operational tests with virtual power plant simulations.

The goal of CCSI is to deliver a toolset that can simulate the scale-up of a broad set of new carbon capture technologies from laboratory scale to full commercial scale. To provide a framework around which the toolset can be developed and demonstrated, we will focus on three Industrial Challenge Problems (ICPs) related to carbon capture technologies relevant to U.S. pulverized coal (PC) power plants. Post combustion capture by solid sorbents is the technology focus of the initial ICP (referred to as ICP A).

The goal of the uncertainty quantification (UQ) task (Task 6) is to provide a set of capabilities to the user community for the quantification of uncertainties associated with the carbon capture processes. As such, we will develop, as needed and beyond existing capabilities, a suite of robust and efficient computational tools for UQ to be integrated into a CCSI UQ software framework.

\subsection{UNCERTAINTY QUANTIFICATION TASK}

The approach of the UQ team will be to leverage, as much as possible, existing state-of-the-art tools to provide UQ capabilities for the suite of simulation tools. Some of these tools are available at the DOE National Laboratories and they include, for example, the DAKOTA framework from Sandia National Laboratories, the PSUADE toolset and UQ Pipeline from Lawrence Livermore National Laboratory, the UQ toolsets from Los Alamos National Laboratory and Pacific Northwest National Laboratories, and other tools available from the UQ community. These frameworks provide various UQ tools such as sampling methods, statistical analysis methods, global optimization routines, response surface methods, and workflow management capability to launch and monitor the large ensembles of calculations used in a statistical evaluation of uncertainty. In cases where existing tools do not provide appropriate capabilities (to be anticipated when we move toward physics-based full scale models), we will identify the gaps and develop new UQ approaches. The result will be an end-to-end UQ engine (integrated framework) for CCSI that allows full UQ studies which is equipped with (a) a variety of adaptive sampling methodologies populating high dimensional uncertainty spaces, (b) the capability to launch and monitor a large ensemble of calculations, and (c) the functionality to collect and analyze the output data.

Specific activities for year 1 (through 1/31/2012) for Task 6 include the following:

6.1 Survey and evaluate UQ methodologies for Carbon Capture process simulators 6.1.1 Compile CCSI process model characteristics 
6.1.2 Compile relevant UQ methodologies and methods

6.1.3 Compile existing UQ tools

6.1.4 Document results from each 6.1 subtasks

6.2 Demonstrate UQ methodology on MEA simulations.

6.2.1 Define UQ objective and available experimental data for MEA

6.2.2 Identify parameters and probability distributions in MEA

6.2.3 Define/implement UQ framework for MEA

6.2.4 Perform UQ studies on MEA

6.2.5 Release UQ framework (version 1) and complete report

This report documents progress on Task 6.2 through the end of January 2012. 


\subsection{UQ Tool's Capabilities}

To perform uncertainty quantification on a model simulating some physical phenomenon, we follow a few key steps:

1) Identify dominant sources of uncertainties including observation data;

2) Characterize the selected sources of uncertainties;

3) Propagate the uncertainties through the model via sampling; and

4) Analyze simulation results to assess uncertainties and sensitivities.

Step (1) involves frequent dialog with subject matter experts (SMEs). For example, in the UQ study of the CCSI MEA model, the UQ team had many sessions with the process design/synthesis team to identify sources of uncertainty that the process designers deemed significant. Our initial focus on the UQ method development is to support uncertainties that can be described by continuous uncertain parameters. Once these uncertain parameters have been identified, Step (2) also involves conversing with SMEs or searching through the literature in order to prescribe ranges (in the form of lower and upper bounds) and probability distributions (e.g. normal, uniform, etc.) for these parameters. UQ methods/tools are needed for Steps (3) and (4). A simple workflow for Steps (3) and (4) is depicted in Figure 2-1.

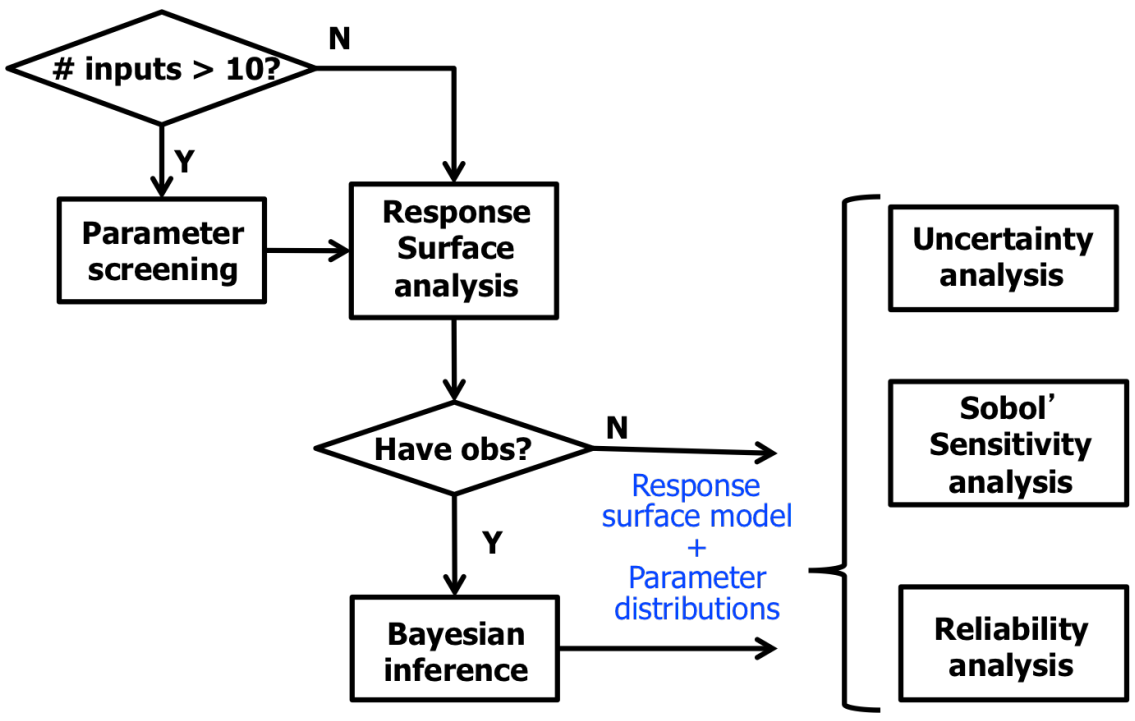

Figure 2-1: Simple workflow for UQ analysis

If the number of input parameters is greater than ten, then it is advisable to apply parameter screening to perform variable selection (to derive smaller subset of "important" input parameters). Once the number of input parameters is more manageable, one can perform response surface analysis to approximate the functional relationship between the input samples and the output samples. If observational data exists for the output parameters, then one could perform Bayesian inference (also known as data fusion, parameter estimation, or inverse UQ) to refine the estimates of the input uncertainties (which may prompt the generation of new samples or filtering of old samples based on the new input uncertainties). Finally, when an adequate characterization of the input uncertainties (in the form of parameter distributions) and the input-to-output mapping (in the form of a validated response surface model) is obtained, then one may proceed with uncertainty analysis, sensitivity analysis, reliability analysis, etc. 
In order to support this workflow, we have developed a graphical user interface (GUI) coupled to the PSUADE UQ software. This integrated UQ engine currently has the following capabilities:

Sample generation: The user specifies a sample size and a sampling scheme. Once specified, the GUI will generate a sample (consisting a number of sample points), then invoke the Aspen Plus client software (developed by Element 5) to evaluate the sample, which would then serve as data to subsequent UQ analyses. Several sampling schemes such as Monte Carlo (MC), Quasi-Monte Carlo (QMC), Latin Hypercube (LH), Orthogonal Array (OA), and the Morris design are currently available through the GUI. The choice of sampling method depends on which analysis is performed. For example, for parameter screening, the Morris or LH designs are recommended. For uncertainty assessment of inexpensive simulations, QMC or LH designs are preferred over MC. For response surface analysis, space-filling designs such as LH, OA or QMC are useful.

Forward uncertainty analysis: The tool will compute the probability distribution of one single output variable and display its sufficient statistics, such as mean, standard deviation, skewness and kurtosis. The distributions will also be displayed graphically.

Inverse UQ (or Bayesian inference): For each output variable, the user specifies an observed value (from physical experiments) with the associated uncertainties (in the form of standard deviation). The tool will launch the Markov Chain Monte Carlo (MCMC) algorithm to compute the posteriors distributions (prior distributions integrated with data) of the uncertain parameters. The distributions will also be displayed graphically.

Response surface model analysis: The user selects a single output and also a response surface fitting scheme. The tool will use the data (i.e., input-output samples) to "fit" a response surface scheme that approximates the inputs-to-output relationship. The model will be cross-validated to derive its errors (i.e., deviations from the model's predicted values against the true values). The tool will plot a histogram of the errors as well as a plot of predicted data vs. actual data, in which a diagonal plot (for example, see right side of Figure 3-11) signifies an adequate combination of the sample and the selected fitting scheme. Once a response surface is generated, the tool also provides ways to visualize the response surface. Current version of our UQ tool provides a few fitting schemes such as polynomial regression and splines. Future versions will add more schemes.

Forward uncertainty analysis using response surface model: In case the current sample is not large enough to give meaningful statistics, the sample can be used to create a response surface, which can be evaluated to efficiently generate a much larger sample, in order to derive an improved estimate of the statistics.

Sample refinement via adaptive sampling: After a sample has been generated, evaluated, and fitted via response surface analysis, the user may find that the sample is not sufficiently large to give an accurate enough fit. Under this circumstance, more sample points may be added to the existing sample. Current version of the tool provides crude capabilities for adaptive sampling refinements. There are two methods to add more samples to the existing ensemble. One is uniform refinement, which doubles the sample size by adding sample points uniformly in the parameter space. The other is adaptive refinement, which will generate a new sample via the METIS sampling scheme. Using this METIS sample, additional sample points will be derived from regions in the uncertain parameter space that corresponds to high variability in the output space. The adaptive refinement method iterates between adding new sample points and evaluating them via simulation. The "appended" sample can be analyzed again using response surface analysis tools to evaluate its adequacy for model fitting.

Variance-based sensitivity analysis: Given a user-specified output, the tool will determine each uncertain variable's contribution to the total variance of the output. The result is displayed as a pie chart 
for clarity. Current version supports first-order sensitivity only. Higher-order sensitivity analysis will be provided in the next version.

Parameter screening: This capability is equivalent to variable selection. In the current version, only the Morris method is supported (thus the sample needs to be generated using the Morris sampling scheme). This is a qualitative sensitivity analysis method that identifies a group of dominant parameters that are recommended for inclusion in subsequent UQ analyses, as they are the ones that most impact the output uncertainty. The results will be displayed so that users can also rank the uncertain parameters visually. More methods will be added in the future.

Scatter plot: The current version supports displaying graphs of a user-specified output against each uncertain parameter.

For details of the statistical theory behind each capability, please refer to the PSUADE Theory Manual (Tong, 2009). 


\subsection{UQ Graphical User Interface}

The current CCSI UQ Toolset/GUI requires Matlab 7.11.0 (2010b), PSUADE 1.4 and Python 2.6+. It was developed and tested on Linux and Macintosh systems; its compatibility with other platforms has not been tested.

The user interacts with the CCSI UQ Toolset via the associated GUI, which in turn calls the PSUADE statistical software to perform sampling and UQ analyses. The GUI is implemented in Matlab and PSUADE is implemented in $\mathrm{C} / \mathrm{C}++/$ Fortran.

The initial UQ Toolset/GUI is tailored for use with Aspen Plus, in particular for running the MEA process models. The tool provides capabilities to communicate with a web-based gateway capable of running large numbers of concurrent Aspen Plus simulators. The communication protocols have been implemented through Python scripts (by the CCSI Integration Framework Team). Thus, while Python is not required for UQ analyses, it is required for any capability involving sample generation, such as sample generation or sample refinement. If the user decides to not install Python, he/she can still perform UQ analyses with existing data (i.e., sample inputs/outputs) stored in PSUADE format.

In the following, we show a few snapshots of the CCSI UQ GUI. Details of how to steer through the GUI can be found in the UQ Toolset/GUI V1 framework user manual (under preparation).

\subsection{MODEL PREPARATION}

When the user starts up the GUI, the following splash screen will appear.

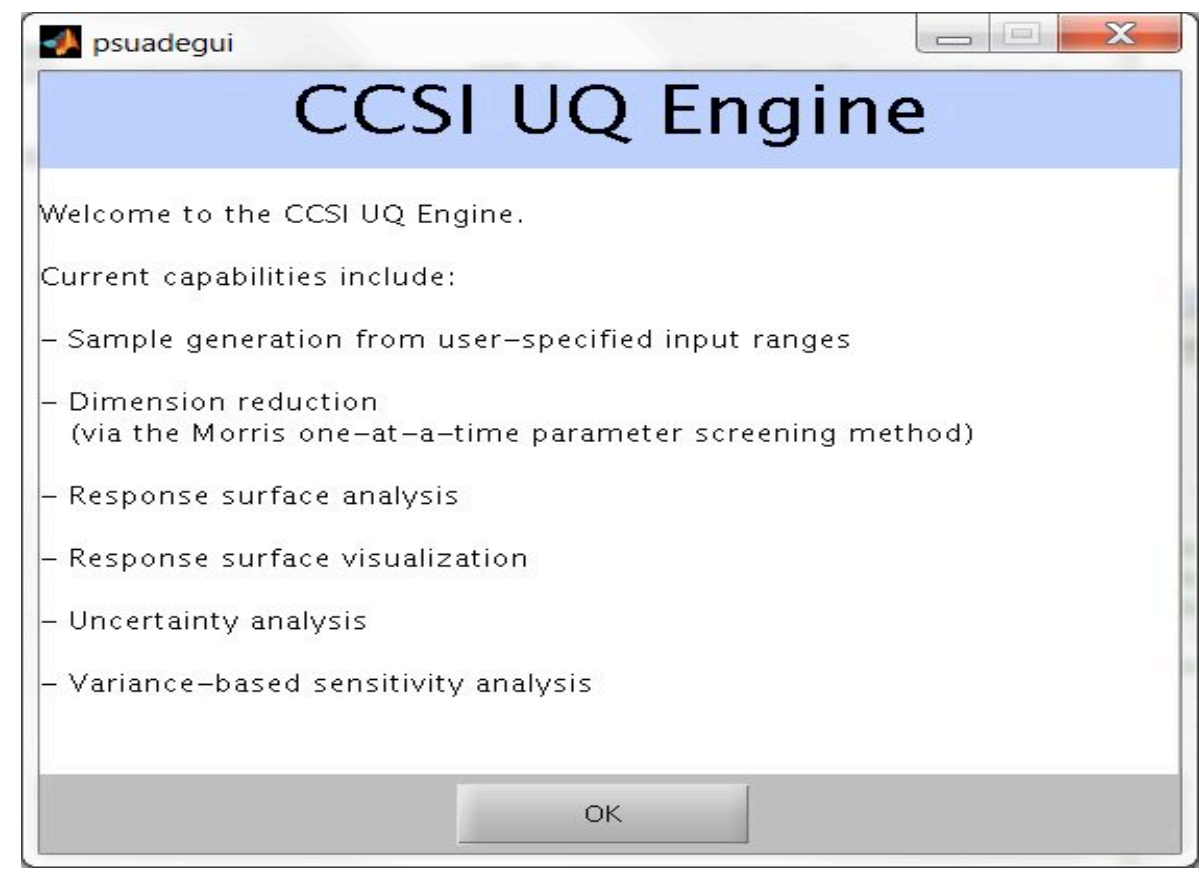

Figure 3-1: GUI splash screen

The next screen (cf. Figure 3-2) allows the user to choose from three options. The user can: configure a set of samples from scratch, load up a previous configuration for editing, or open a set of previously generated samples for execution and/or analysis. In the first two cases, the user may configure samples as described in the next section. If the user elects to load a set of pre-generated samples, the user is sent directly to the Run/Analysis screen, as described in Section 3.5. 


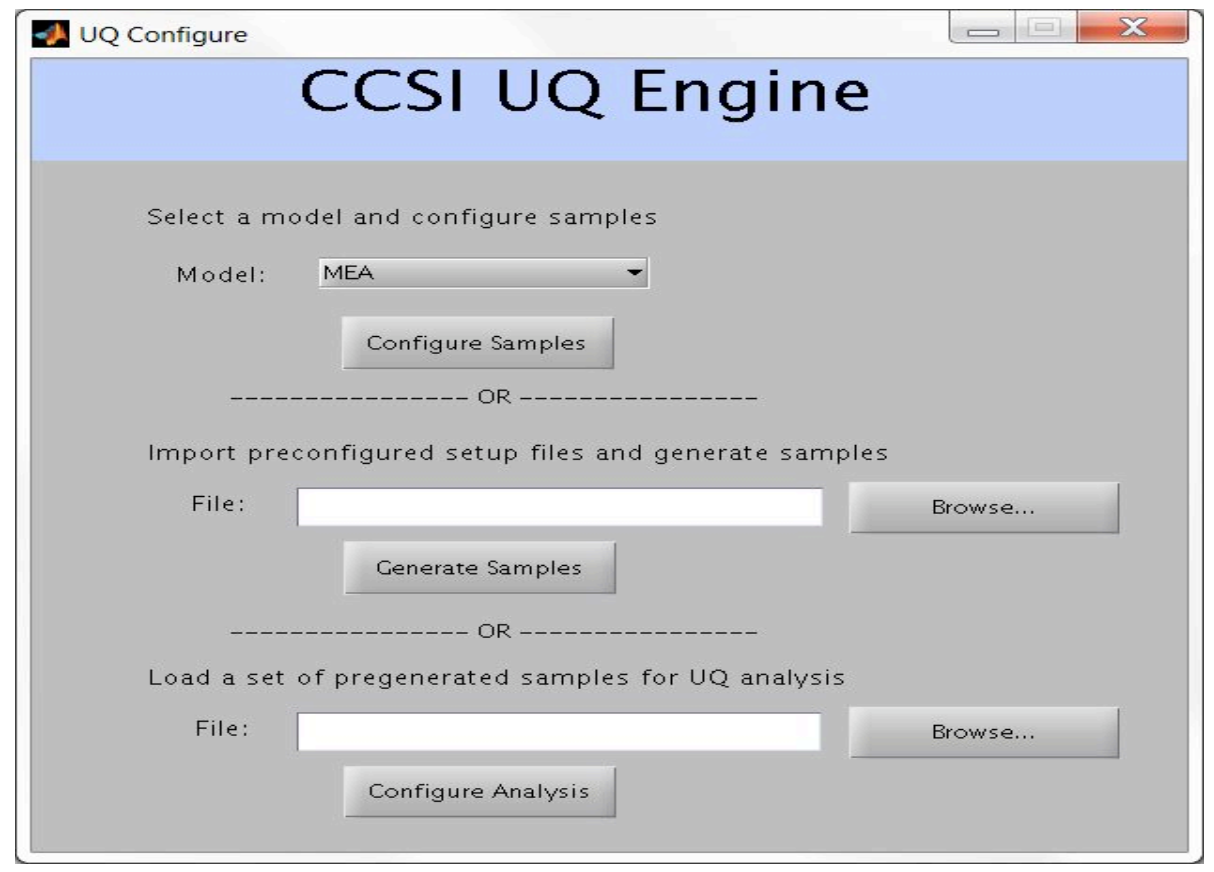

Figure 3-2: Configuration/load screen

\subsection{SAMPLE CONFIGURATION}

To generate new samples, the user needs to select a model (e.g., MEA). The GUI will then read the simulation configuration file and display a tabbed interface that allows the user to configure the samples, including selection of inputs and outputs of interest.

On the inputs tab (cf. Figure 3-3), the user must select the input(s) that he/she wishes to vary. The user needs to define a range for each selected input. The user may also specify a probability distribution (such as the normal distribution) that effectively characterizes that input's uncertainty (the distribution is uniform by default). 


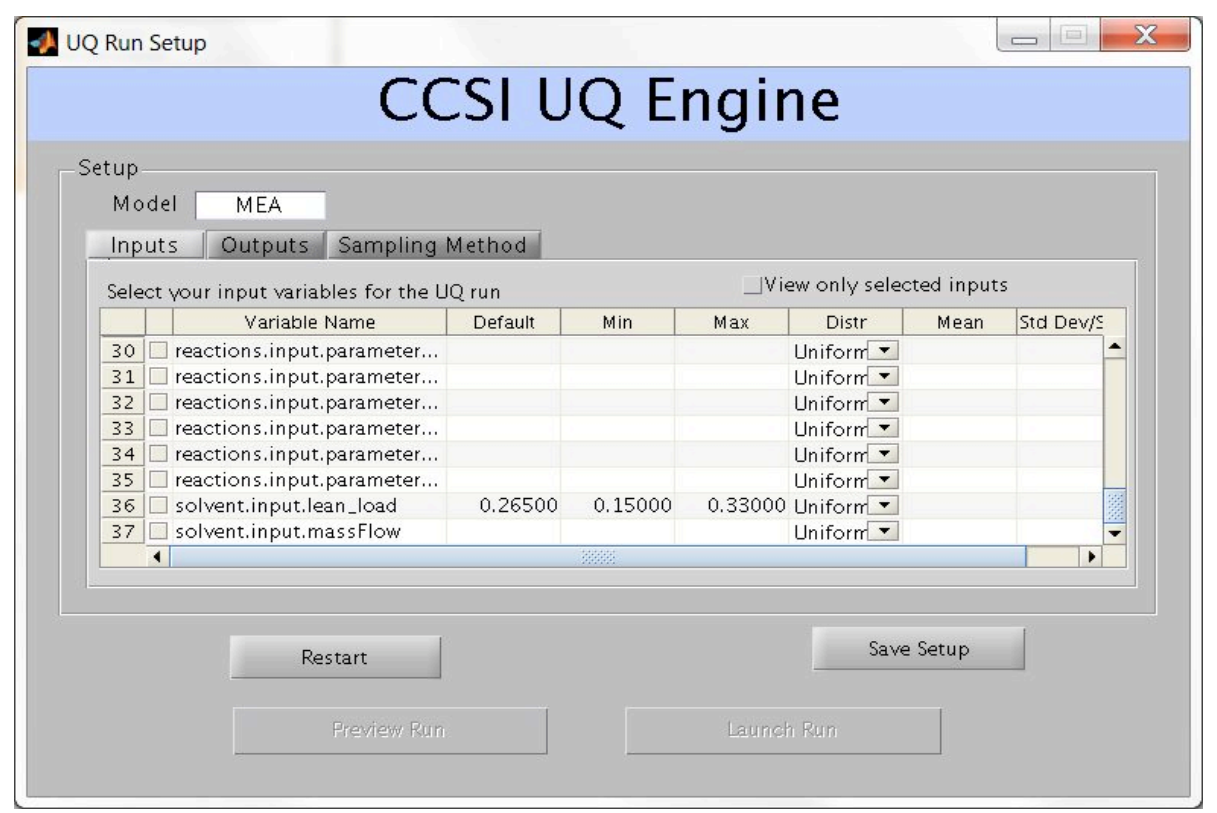

Figure 3-3: Input selection screen

On the outputs tab (cf. Figure 3-4), the user must select the output(s) that he/she wishes to analyze.

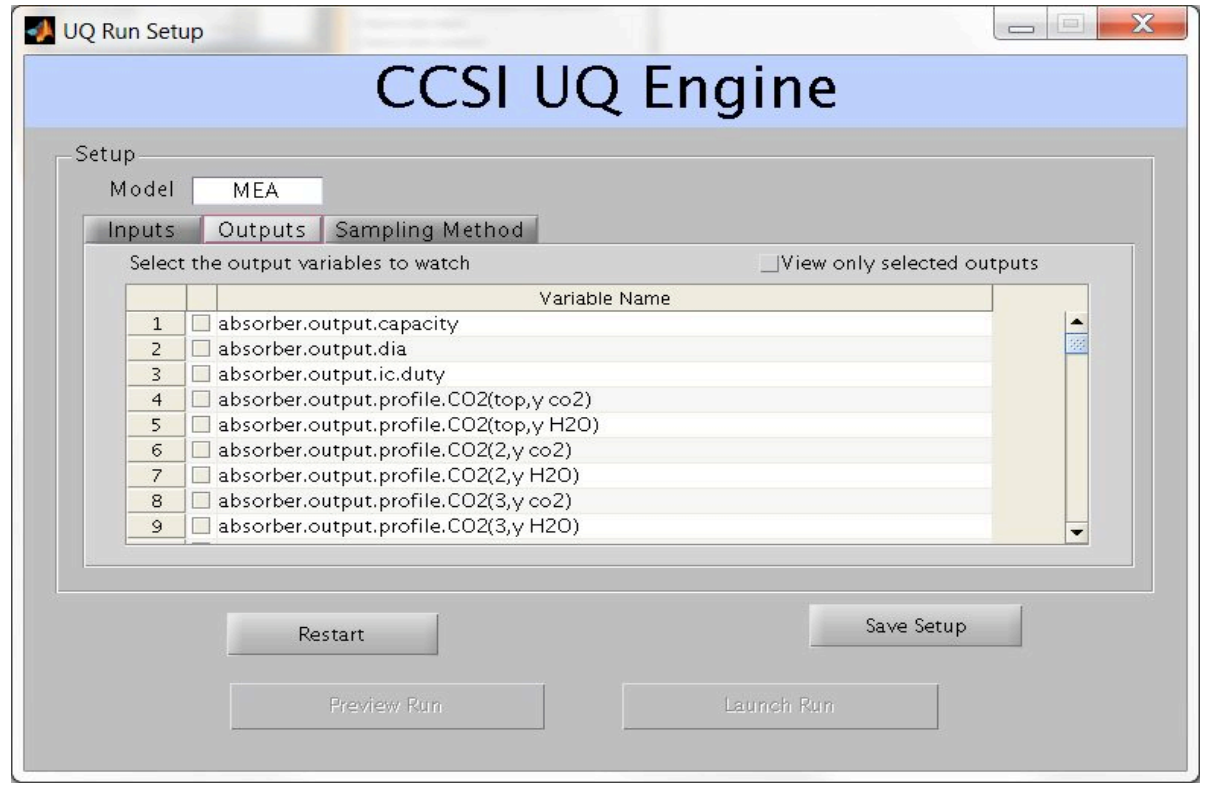

Figure 3-4: Output selection screen

On the sampling method tab (cf. Figure 3-5), the user may also select the sampling method to use for generating the input samples, as well as the number of samples to generate. 


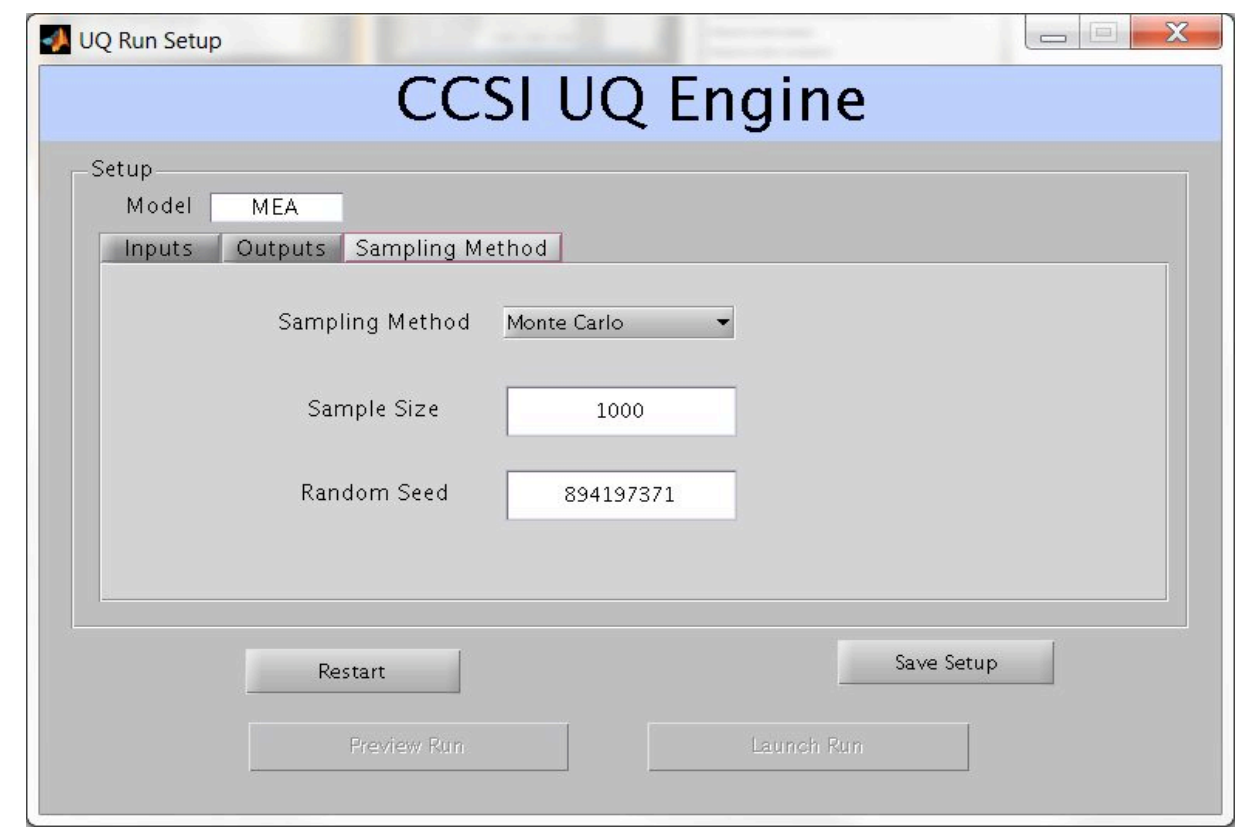

Figure 3-5: Sampling scheme selection screen

\subsection{SAMPLE GENERATION}

Once the user has configured the samples to his/her satisfaction, the user may preview the samples or immediately start running the simulations. Either way, the GUI will generate a PSUADE file with information about the input/output variables and the sampling scheme, then invoke PSUADE to generate the input samples. If the user has opted to preview the samples, the screen in Figure 3-6 will appear. This screen allows the user to verify that the samples match his/her expectations, and to make any necessary modifications to the samples. 


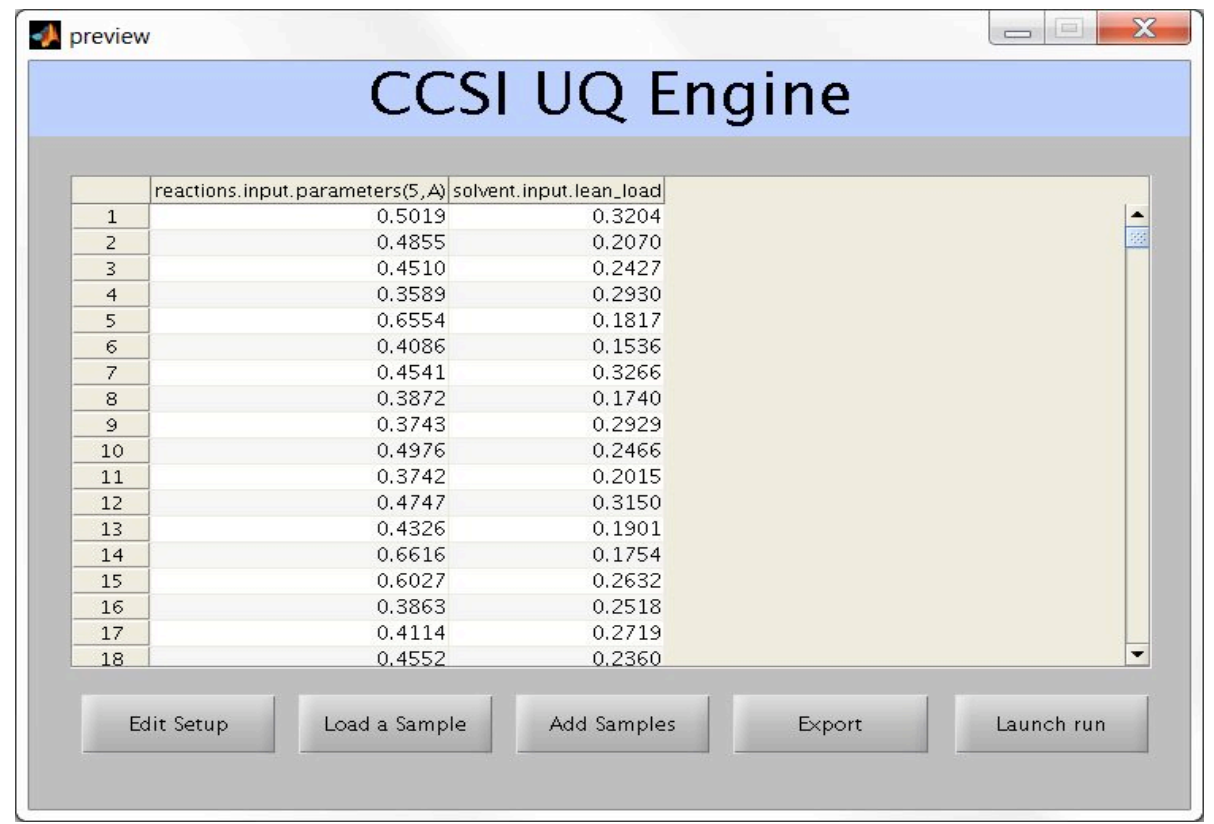

Figure 3-6: Sample preview screen

\subsection{LAUNCHING SIMULATION RUNS}

The user may click "Launch Run" from either one of the sample configuration or sample preview screens. This will bring up the Run/Analysis screen in Figure 3-7. At this point, the user may click "Run" to actually launch the runs. The GUI will then pass the samples to the Python scripts for submission to the gateway. The GUI will monitor progress at the gateway, and as the runs complete, the progress bar will change from blue to red.

The simulations could take a long time, so the GUI provides options for the user to stop and resume the simulation runs. If the user stops the runs, the GUI will retrieve the partial results from the completed runs. Whether the user allows the runs to complete, or the user stops the runs when they are partially completed, the user will still be able to do analysis on the samples that have completed and been retrieved by the GUI.

\subsection{UQ ANALYSIS}

Once the sample runs are complete or existing samples are loaded, the user will see this screen in which he/she can choose a specific UQ method for analysis from the pull-down menu. All capabilities described in Section 2 can be selected from this menu. Here, we demonstrate a subset of these capabilities. 


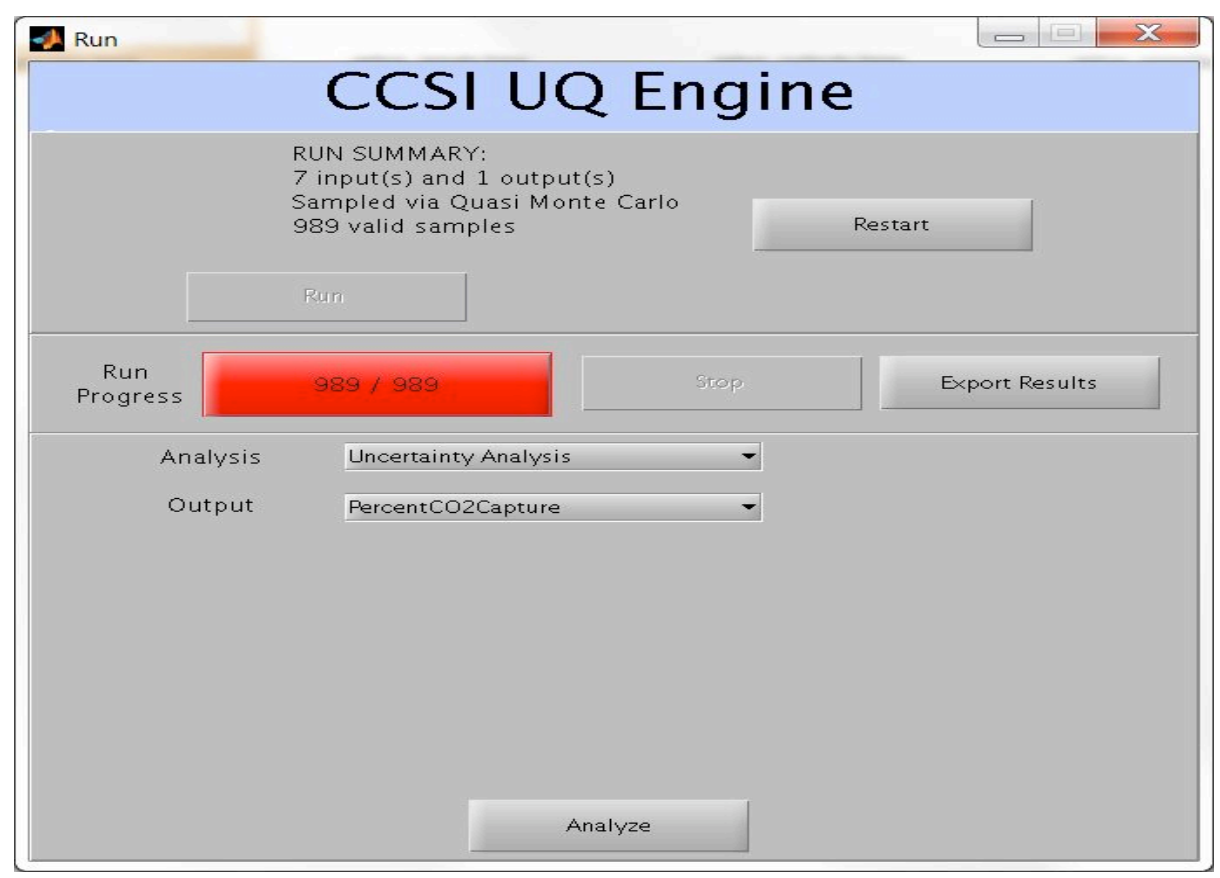

Figure 3-7: Sample generation progress and UQ method selection screen

\section{Uncertainty Analysis}

The user might be interested in the output uncertainty resulting from the input uncertainties. By choosing "Uncertainty Analysis", one may view the PDF and sample statistics of the selected output variable.

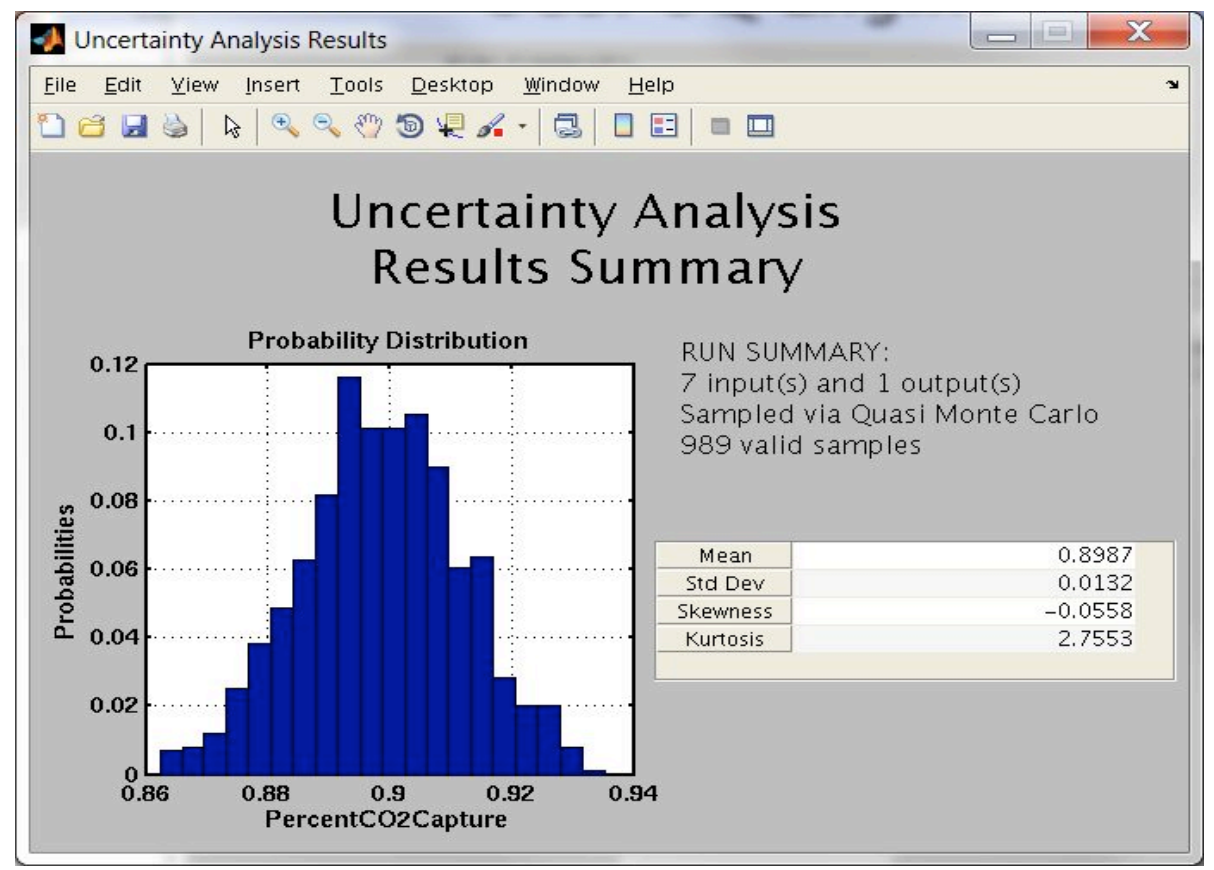

Figure 3-8: Uncertainty analysis results screen 


\section{Parameter Screening}

The user may also wish to determine which input variables have the most impact on the output variables. There are multiple ways to do this. A simple method is to examine the scatter plots of the selected output against each of the uncertain parameters. These scatter plots are very useful in understanding the trend of the output when the inputs are varied. The inputs with obvious upward/downward trends are important while the ones with no trends are relatively unimportant.

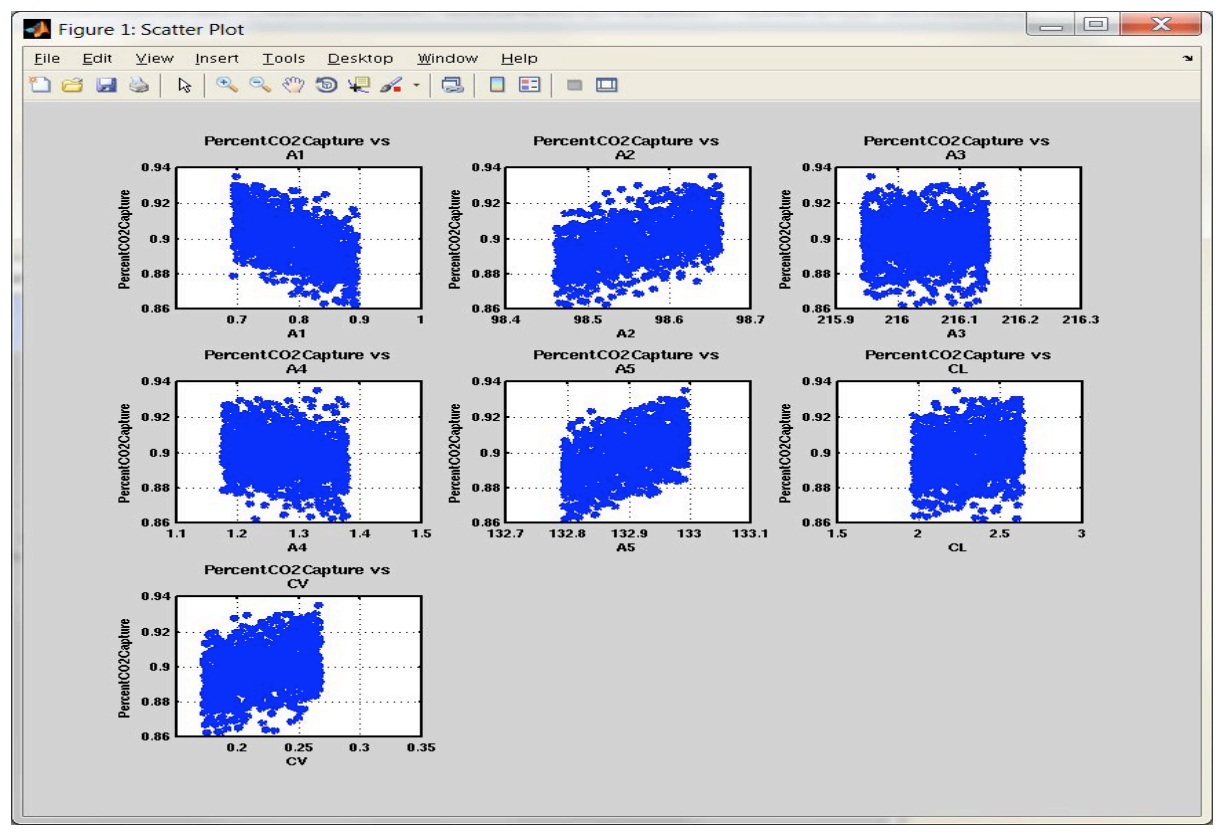

Figure 3-9: Scatter plot screen

Another, more quantitative, way to discern the importance of a specific input is to perform variance-based sensitivity analysis. This shows the apportioning of the output variability to individual input parameters by percentage.

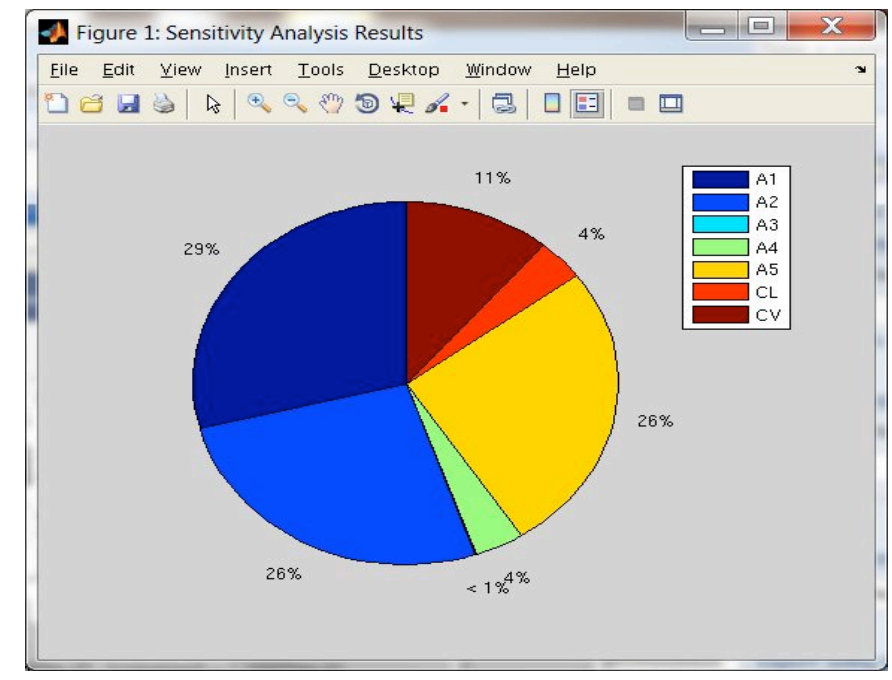

Figure 3-10: Variance-based sensitivity analysis screen 


\section{Response Surface Analysis \& Visualization}

The user may also find it useful to approximate the input-to-output mapping with a response surface. The GUI provides multiple options for response surface generation, including multivariate adaptive regression splines and linear/quadratic/cubic regression. (More options will be included in future versions.) To help the user decide which response surface model offers the best fit, the user may select "Validate Response Surface" for the GUI to display validation plots that show deviations from the response surface's predicted values against the actual values. Here, this specific response surface appears to be a good fit for the data.

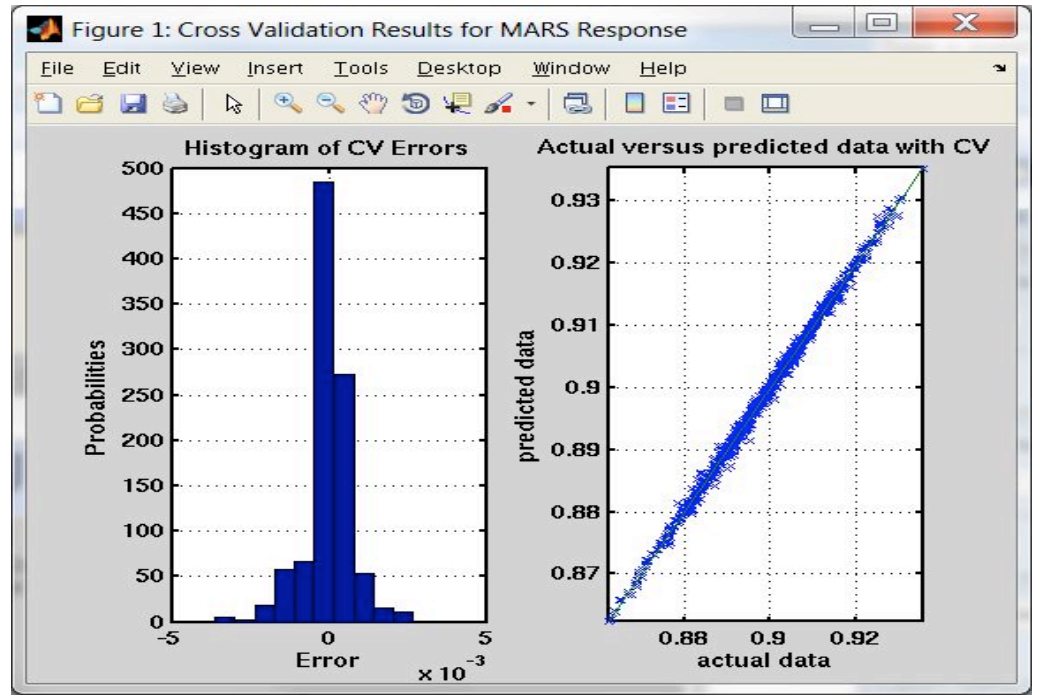

Figure 3-11: Response surface validation screen

Once the user is content with the specific choice of response surface, the user can use the GUI to visualize the response surface that maps from the most important inputs to a single output. Currently, 1-D, 2-D and 3-D visualization is supported. Figure 3-12 shows a sample 2-D response surface.

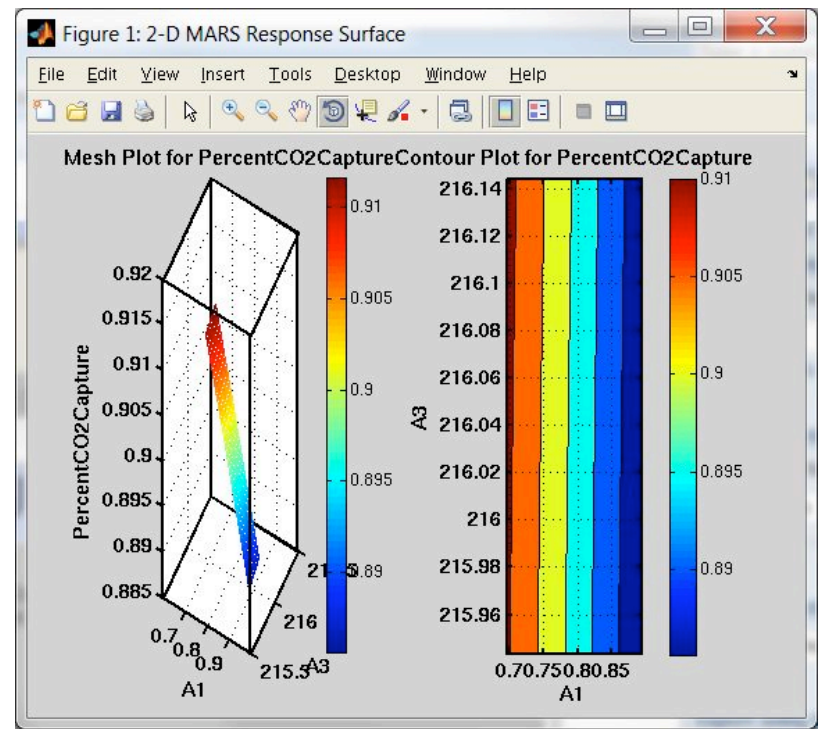

Figure 3-12: Response surface visualization screen 


\subsection{MEA Process Models}

We applied the CCSI UQ Toolset to study the uncertainties in two MEA models provided by the Process Synthesis \& Design Team. The two models were implemented within Aspen Plus; each represents a model of the absorber column with different assumptions on the chemical reactions and mass transfer correlations. The first model assumed chemical reactions at equilibrium and used the Billet and Schultes correlation (Billet and Schultes, 1993), while the second model assumed a mixture of equilibrium and rate-based reactions and used the Hanley structured correlation (Hanley and Chen, 2012).

\subsection{MODEL V1}

\section{Chemical reactions}

There are many chemical reactions that occur within the absorber, the simplest of which are the ones in dynamic equilibrium. A chemical reaction in equilibrium is one in which the concentrations of the reactants and products have no net change in time. If a reaction is allowed time to reach equilibrium, then theoretically the equilibrium concentrations of the reactants and products can be measured and combined as a ratio, known as the equilibrium constant.

For example, in the equilibrium reaction where reactants $\mathrm{X}$ and $\mathrm{Y}$ react to produce products $\mathrm{P}$ and $Q$, and vice versa:

$$
X+Y \leftrightarrow P+Q
$$

the equilibrium constant is:

$$
K=\frac{[P]^{p}[Q]^{q}}{[X]^{x}[Y]^{y}}
$$

where the exponents $\mathrm{x}, \mathrm{y}, \mathrm{p}$, and $\mathrm{q}$ are orders which depend on the reaction mechanism; and $[\mathrm{X}]$ represents the concentration of substance $\mathrm{X}$ in moles per volume of solution.

The equilibrium constant is insensitive to initial concentrations of reactants and products, but $i s$ sensitive to temperature and pressure. However, since the pressure dependence is weak within the ranges of pressured deployed in industry, this dependence is often ignored in practice.

The five equilibrium reactions pertinent to Model V1 are:

\begin{tabular}{|c|c|c|c|}
\hline $\mathrm{H}_{2} \mathrm{O}+\mathrm{MEAH}^{+}$ & $\leftrightarrow$ & $\mathrm{H}_{3} \mathrm{O}+\mathrm{MEA}$ & (Reaction V1-1) \\
\hline $\mathrm{CO}_{2}+\mathrm{OH}^{-}$ & $\leftrightarrow$ & $\mathrm{HCO}_{3}^{-}$ & (Reaction V1-2) \\
\hline $\mathrm{H}_{2} \mathrm{O}+\mathrm{HCO}_{3}^{-}$ & $\leftrightarrow$ & $\mathrm{H}_{3} \mathrm{O}^{+}+\mathrm{CO}_{3}^{2-}$ & (Reaction V1-3) \\
\hline $\mathrm{MEACOO}^{-}+\mathrm{H}_{2} \mathrm{O}$ & $\leftrightarrow$ & $\mathrm{MEA}+\mathrm{HCO}_{3}^{-}$ & (Reaction V1-4) \\
\hline $2 \mathrm{H}_{2} \mathrm{O}$ & $\leftrightarrow$ & $\mathrm{H}_{3} \mathrm{O}^{+}+\mathrm{OH}^{-}$ & (Reaction V1-5) \\
\hline
\end{tabular}

Table 4-1: Equilibrium reactions from Aspen MEA insert

There exists a relationship between equilibrium constants and temperature:

$$
\ln K=A+\frac{B}{T}+C \ln T+D T
$$


where $T$ is the temperature. Within the Aspen Plus environment, the parameters A, B, C, and D are modifiable inputs. The nominal values for these parameters are set to:

Table 4-2: Equilibrium constant parameters from Aspen MEA insert

\begin{tabular}{ccccc}
\hline Reaction & A & B & C & D \\
\hline V1-1 & 0.7996 & -8094.81 & 0.0 & -0.007484 \\
V1-2 & 98.566 & 1353.8 & -14.3043 & 0.0 \\
V1-3 & 216.049 & -12431.7 & -35.4819 & 0.0 \\
V1-4 & 1.282562 & -3456.179 & 0.0 & 0.0 \\
V1-5 & 132.899 & -13445.9 & -22.4773 & 0.0 \\
\hline
\end{tabular}

For Model V1, we assumed uncertainty surrounding only the A parameters while keeping all other parameters fixed at their nominal values. Our SMEs had recommended that a uniform distribution over a $+/-10 \%$ interval centered on the nominal value for $\mathrm{A}$.

\section{Mass transfer correlation}

Another important aspect of the absorber is the model of the distillation column, which contains random or structured packings to facilitate the countercurrent contacting of liquid with gas. The main variables that affect the performance of these packed columns are: liquid holdup, pressure drop, maximum flow capacity, and mass-transfer efficiency. In this study, we focused on the mass transfer coefficients and the effective interfacial area, which are parameters that affect the mass-transfer efficiency. There are two mass transfer coefficients - one for liquid phase and another for vapor phase. Each describes the rate of mass transfer per unit interfacial area per unit concentration difference across the contact surface where equilibrium is assumed.

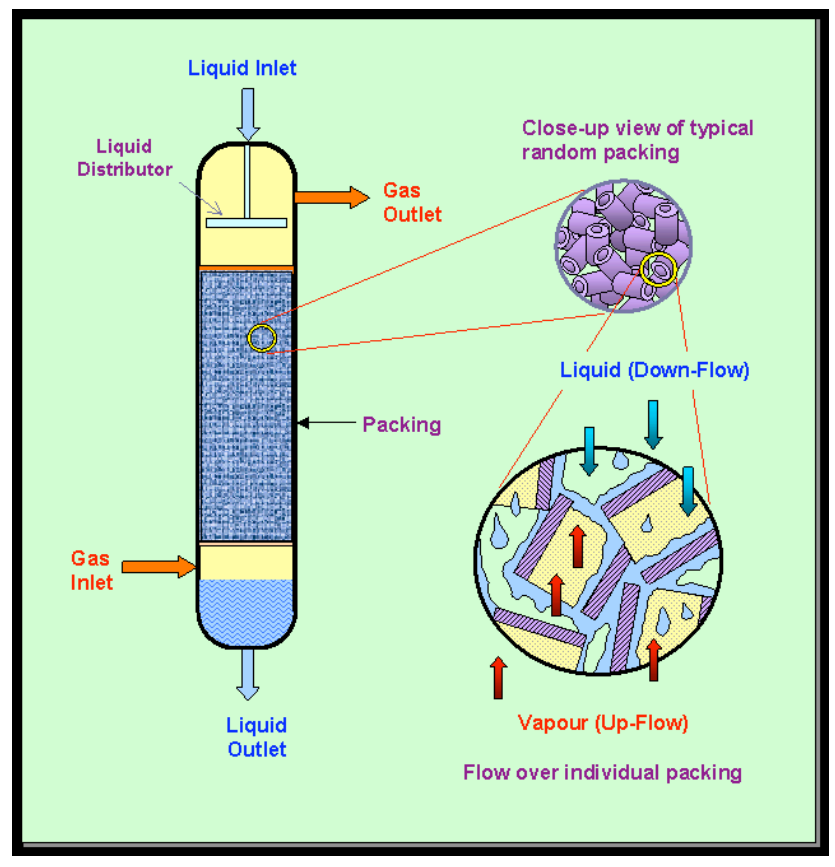

Figure 4-1: Schematic of a packed column

[Source: separationprocesses.com] 
Traditionally, the mass-transfer performance of a packed column is described by the packed bed height equivalent to a theoretical plate (HETP). The HETP is the height of packing in a distillation column that gives a separation equivalent to one theoretical stage, defined as the contact process between vapor and liquid such that the exiting vapor and liquid streams are in equilibrium. It is given by the expression:

$$
H E T P=\frac{\ln \lambda}{\lambda-1}\left(\frac{u_{V}}{k_{V} a_{e}}+\lambda \frac{u_{L}}{k_{L} a_{e}}\right)
$$

where $\lambda$ is the stripping factor (i.e., the ratio of the slope of the equilibrium line to that of the operating line), $u_{V}$ and $u_{L}$ are the vapor- and liquid-phase superficial velocities, $k_{V}$ and $k_{L}$ are the vapor- and liquid-phase mass-transfer coefficients, and $a_{e}$ is the effective interfacial area provided by the packing to enable mass transfer.

While there are many models of mass-transfer correlations for packed columns ${ }^{1}$, Model V1 uses the Billet and Schultes correlation ${ }^{2}$ for structured packings:

$$
k_{L}=C_{L}\left(\frac{g \rho_{L}}{\mu_{L}}\right)^{0.167} \sqrt{\frac{D_{L}}{d_{k}}}\left(\frac{u_{L}}{a_{p}}\right)^{0.333} \quad k_{V}=C_{V}\left(\frac{1}{\sqrt{\varepsilon-h_{t}}}\right) \sqrt{\frac{a_{p}}{d_{k}}} D_{V} R e_{V}^{0.75} S c_{V}^{1 / 3}
$$

where $C_{L}$ is the liquid mass transfer coefficient parameter and $C_{V}$ is the vapor mass transfer coefficient parameter, both of which are characteristic of the shape and structure of the packing.

\begin{tabular}{|c|c|c|}
\hline Terms in $k_{L}$ & Terms in $k_{V}$ & Terms in both \\
\hline $\begin{array}{l}\text { - } g \text { : gravitational acceleration } \\
\left(\mathrm{m} / \mathrm{s}^{2}\right) \\
\text { - } \rho_{L}: \text { density of the liquid }\left(\mathrm{kg} / \mathrm{m}^{3}\right) \\
\text { - } \mu_{L}: \text { viscosity of the liquid }(\mathrm{kg} / \mathrm{m} \\
\mathrm{s}) \\
\text { - } D_{L}: \text { diffusivity of the liquid } \\
\left(\mathrm{m}^{2} / \mathrm{s}\right) \\
\text { - } u_{L}: \text { superficial velocity of the } \\
\text { liquid }(\mathrm{m} / \mathrm{s})\end{array}$ & $\begin{array}{l}\text { - } \varepsilon \text { : void fraction of the } \\
\text { packing } \\
\text { - } h_{t}: \text { fractional holdup } \\
\text { - } D_{V}: \text { diffusivity of the vapor } \\
\left(\mathrm{m}^{2} / \mathrm{s}\right) \\
\text { - } R e_{V}: \text { Reynolds number for } \\
\text { the vapor } \\
\text { - } S c_{V}: \text { Schmidt number for the } \\
\text { vapor }\end{array}$ & $\begin{array}{l}\text { - } a_{p}: \text { specific area of the } \\
\text { packing }\left(\mathrm{m}^{2} / \mathrm{m}^{3}\right) \\
-d_{k}: \text { hydraulic diameter }(\mathrm{m})\end{array}$ \\
\hline
\end{tabular}

Table 4-3: Terms in the Billet and Schultes correlation

For this correlation, we restricted our attention to the mass transfer coefficients, $k_{L}$ and $k_{V}$. Our assessment of their uncertainties is from published experimental results in (Billet and Schultes,

\footnotetext{
${ }^{1}$ Please refer to (Wang et al., 2005) for a comprehensive review.

${ }^{2}$ Equations taken from Aspen's documentation on the Billet and Schultes correlation.
} 
1993), which indicate a standard deviation of $8.3 \%$ for $k_{L}$ and a standard deviation of $12.4 \%$ for $k_{V}$.

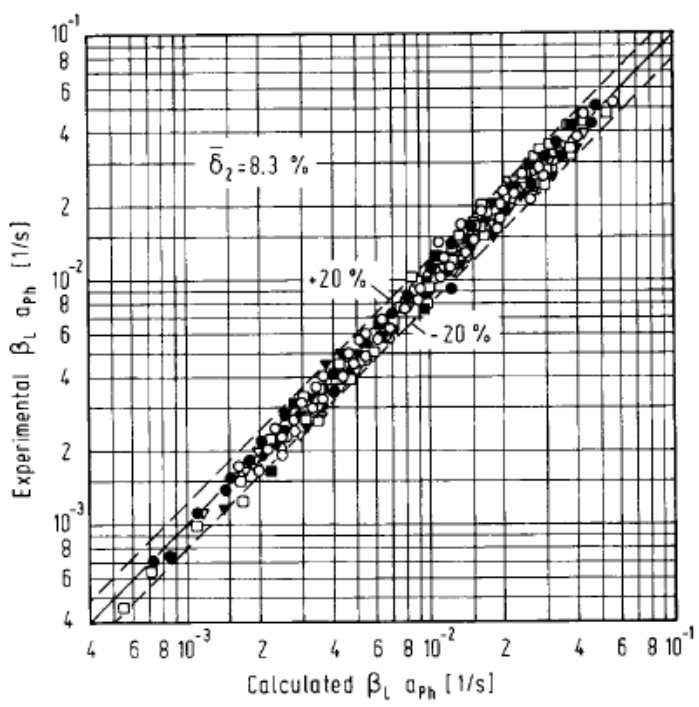

Fig. 1. Comparison of the volumetric mass transfer coefficient in the liquid phase, calculated from Eq. (6), with experimental results.

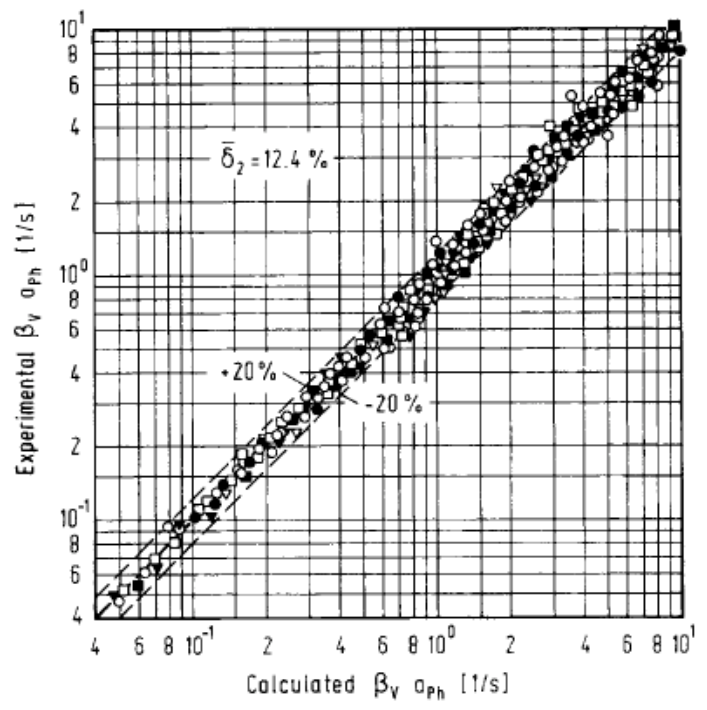

Fig. 2. Comparison of the volumetric mass transfer coefficient in the gas phase, calculated from Eq. (10), with experimental results.

Figure 4-2: Experimental data from (Billet \& Schultes, 1993)

Since $C_{L}$ and $C_{V}$ lend themselves as convenient scaling parameters and are modifiable inputs within the Aspen Plus environment, we attributed uniform distributions over $C_{L}$ and $C_{V}$ using default Aspen value as the mean and the standard deviations of $8.3 \%$ and $12.4 \%$, while holding all other terms constant at their nominal values.

\subsection{MODEL V2}

\section{Chemical reactions}

In this model, all reactions are assumed to be in chemical equilibrium except those of $\mathrm{CO}_{2}$ with $\mathrm{OH}^{-}$and $\mathrm{CO}_{2}$ with MEA. Each of these rate-based, non-equilibrium reactions is associated with a reaction rate, which measures how the speed at which that particular reaction takes place.

For example, in the forward reaction where reactants $\mathrm{X}$ and $\mathrm{Y}$ react to produce products $\mathrm{P}$ and Q:

$$
X+Y \rightarrow P+Q
$$

the rate of reaction $\mathrm{r}$ is related to the reactants' concentrations:

$$
r=k(T)[X]^{x}[Y]^{y}
$$

where $\mathrm{k}(\mathrm{T})$ represents the reaction rate coefficient (usually in units of mole $\mathrm{m}^{-3} \mathrm{~s}^{-1}$ ). 
The reaction rate coefficient $\mathrm{k}(\mathrm{T})$ is dependent on the temperature, as quantified by the Arrhenius equation:

$$
k=\alpha e^{-\frac{E}{R T}}
$$

where $\alpha$ is the pre-exponential factor, $\mathrm{E}$ is the activation energy and $\mathrm{R}$ is the ideal gas constant.

Let $k_{+}$be the reaction rate coefficient for the forward reaction:

$$
X+Y \rightarrow P+Q
$$

and $k_{-}$be the reaction rate coefficient for the backward reaction:

$$
P+Q \rightarrow X+Y
$$

When the two reactions are in equilibrium:

$$
X+Y \leftrightarrow P+Q
$$

the rates of the forward and backward reactions are equal:

$$
k_{+}[X]^{x}[Y]^{y}=k_{-}[P]^{p}[Q]^{q}
$$

Moreover, the ratios of reaction rate coefficients is equivalent to the equilibrium constant:

$$
K=\frac{k_{+}}{k_{-}}=\frac{[P]^{p}[Q]^{q}}{[X]^{x}[Y]^{y}}
$$

The four rate-based reactions in Model V2 are:

Table 4-4: Kinetic reactions from Aspen MEA-REA insert

\begin{tabular}{rlr}
\hline $\mathrm{CO}_{2}+\mathrm{OH}^{-}$ & $\rightarrow \mathrm{HCO}_{3}^{-}$ & (Reaction V2-4) \\
$\mathrm{HCO}_{3}^{-}$ & $\rightarrow \mathrm{CO}_{2}+\mathrm{OH}^{-}$ & $($Reaction V2-5) \\
$\mathrm{MEA}+\mathrm{CO}_{2}+\mathrm{H}_{2} \mathrm{O}$ & $\rightarrow \mathrm{MEACOO}^{-}+\mathrm{H}_{3} \mathrm{O}^{+}$ & $($Reaction V2-6) \\
$\mathrm{MEACOO}+\mathrm{H}_{3} \mathrm{O}^{+}$ & $\rightarrow \mathrm{MEA}+\mathrm{CO}_{2}+\mathrm{H}_{2} \mathrm{O}$ & $($ Reaction V2-7) \\
\hline
\end{tabular}

Within the Aspen Plus environment, each rate-based reaction can be set with customized inputs for the pre-exponential factor $\alpha$ and the activation energy E. For our UQ study, we treated $\alpha$ as uncertain and kept $\mathrm{E}$ at its nominal value.

For Reactions (V2-1) and (V2-2), (Pinsent et al., 1956) reported a maximum error of $11 \%$ for the reaction rate coefficient $\mathrm{k}$ using regressed values for $\alpha$ and $\mathrm{E}$ (the same, if not similar, values are adopted by Aspen Plus). Thus, assuming $\mathrm{k}$ is uncertain $+/-11 \%$ around its nominal value, we attributed a uniform distribution over a $+/-11 \%$ interval centered on the nominal value for $\alpha$. 
For Reactions (V2-3) and (V3-4), (Hikita et al., 1977) reported an average deviation of 3.5\% for the reaction rate coefficient $\mathrm{k}$ using regressed values for $\alpha$ and E. Similarly, we attributed $\alpha$ with a uniform distribution using the nominal value as the mean and the average deviation of $3.5 \%$.

As for the equilibrium reactions in Model V2, each reaction's equilibrium constant is no longer parametrized by $\{\mathrm{A}, \mathrm{B}, \mathrm{C}, \mathrm{D}\}$ as in Model V1. Instead, it is parametrized by the "temperature approach" $\Delta \mathrm{T}$, which is the change in temperature. The van't Hoff equation relates the change in temperature to the change in equilibrium constant, and is frequently used to estimate a new equilibrium constant $K_{2}$ at a new absolute temperature $\mathrm{T}_{2}=\mathrm{T}_{1}-\Delta \mathrm{T}$ :

$$
\ln \left(\frac{K_{2}}{K_{1}}\right)=\frac{-\Delta H^{\Theta}}{R}\left(\frac{1}{T_{2}}-\frac{1}{T_{1}}\right)
$$

where $K_{1}$ is the equilibrium constant at temperature $T_{1}, \Delta H^{\Theta}$ is the standard enthalpy of formation, and $\mathrm{R}$ is the ideal gas constant.

The three equilibrium reactions in Model V2 are:

Table 4-5: Equilibrium reactions from Aspen MEA-REA insert

\begin{tabular}{rlll}
\hline $\mathrm{H}_{2} \mathrm{O}+\mathrm{MEAH}^{+}$ & $\leftrightarrow$ & $\mathrm{H}_{3} \mathrm{O}+\mathrm{MEA}$ & \\
$\mathrm{H}_{2} \mathrm{O}+\mathrm{HCO}_{3}^{-}$ & $\leftrightarrow$ & $\mathrm{H}_{3} \mathrm{O}^{+}+\mathrm{CO}_{3}^{2-}$ & (Reaction V2-1) \\
$2 \mathrm{H}_{2} \mathrm{O}$ & $\leftrightarrow$ & $\mathrm{H}_{3} \mathrm{O}^{+}+\mathrm{OH}^{-}$ & (Reaction V2-2) \\
\hline
\end{tabular}

To compute the temperature approach $\Delta \mathrm{T}$ for each of these reactions, we imposed that $\mathrm{K}_{2}$ is within $+/-10 \%$ of $\mathrm{K}_{1}$ (this was the same assumption applied to $\mathrm{K}$ in Model V1), then computed $\Delta \mathrm{T}$ accordingly using the average temperature in the absorber column as $\mathrm{T}_{1}$.

\section{Mass transfer correlation}

While Model V1 uses the Billet and Schultes correlation, Model V2 uses the Hanley correlation ${ }^{3}$ for MELLAPAK/FLEXIPAC sheet metal structured packings:

$$
k_{L}=0.33 R e_{L}^{1} S c_{L}^{1 / 3}\left(\frac{c_{L} D_{L}}{d_{e}}\right) \quad k_{V}=0.0084 R e_{V}^{1} S c_{V}^{1 / 3}\left(\frac{c_{V} D_{V}}{d_{e}}\right)\left(\frac{\cos (\theta)}{\cos (\pi / 4)}\right)^{-7.15}
$$

\footnotetext{
${ }^{3}$ Equations taken from (Hanley and Chen, 2012).
} 
Table 4-6: Terms in the Hanley correlation

\begin{tabular}{|l|l|c|}
\hline Terms in $k_{L}$ & Terms in $k_{V}$ & Terms in both \\
\hline - $R e_{L}:$ liquid-phase Reynolds number & $\bullet R e_{V}:$ vapor - phase Reynolds number & $\bullet d_{e}$ : equivalent \\
- $S c_{L}:$ liquid-phase Schmidt number & $\bullet S c_{V}:$ vapor - phase Schmidt number & diameter $(\mathrm{m})$ \\
- $c_{L}:$ liquid-phase molar concentration & $\bullet c_{V}:$ vapor - phase molar concentration & \\
$\left(\mathrm{mol} / \mathrm{m}^{3}\right)$ & $\left(\mathrm{mol} / \mathrm{m}^{3}\right)$ & \\
- $D_{L}:$ liquid-phase binary diffusivity & $\bullet D_{V}:$ vapor-phase binary diffusivity & \\
$\left(\mathrm{m}^{2} / \mathrm{s}\right)$ & $\left(\mathrm{m}^{2} / \mathrm{s}\right)$ & \\
& $\bullet \theta:$ corrugation inclination angle $(\mathrm{rad})$ & \\
\hline
\end{tabular}

Published experimental results in (Hanley and Chen, 2012) indicate a $+/-10 \%$ error between the predicted HETP values for the correlation and the experimental values.

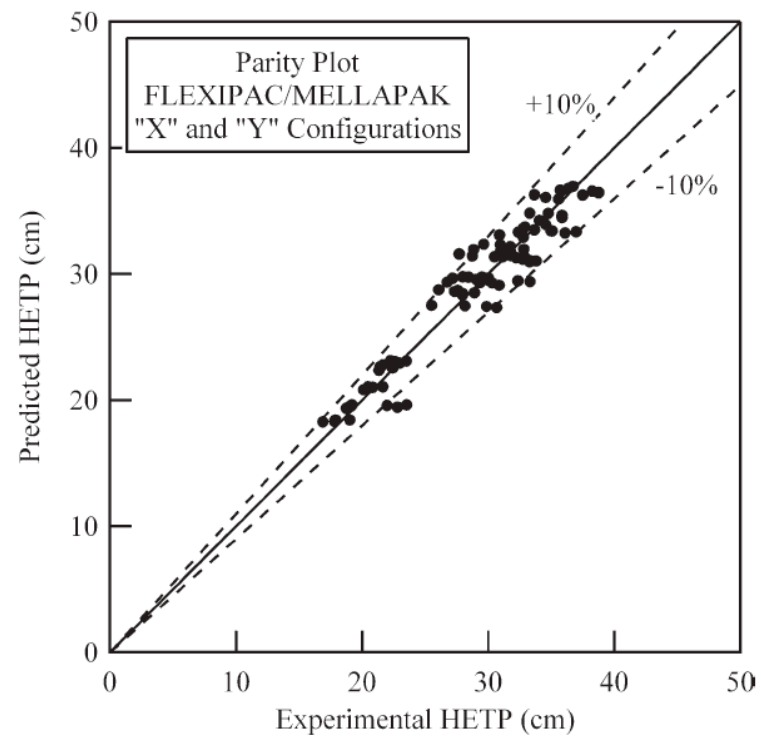

Figure 6. Parity plot comparing the calculated $\langle$ HETP $\rangle$ to the experimentally measured $\langle$ HETP $\rangle$ for sheet metal structured packings of the MELLAPAK/FLEXIPAC type.

Figure 4-3: Experimental data from (Hanley and Chen, 2012)

Unlike the Billet and Schultes correlation from Model V1, there are no parameters that can change the values of $k_{L}$ and $k_{V}$ directly. But since the effective interfacial area $\mathrm{a}_{\mathrm{e}}$ is a modifiable input and it scales the effect of $k_{L}$ and $k_{V}$ in the HETP:

$$
H E T P=\frac{\ln \lambda}{\lambda-1}\left(\frac{u_{V}}{k_{V} a_{e}}+\lambda \frac{u_{L}}{k_{L} a_{e}}\right)
$$


we can indirectly impose a $+/-10 \%$ uncertainty on $k_{L}$ and $k_{V}$ by attributing a uniform distribution over on $\mathrm{a}+/-10 \%$ interval centered on the nominal value for $\mathrm{a}_{\mathrm{e}}$. 


\subsection{UQ Analyses on MEA Process Data}

After having identified the uncertain inputs, we generated samples from each model and applied the UQ Toolset/GUI to perform UQ analyses. We repeated this process for three separate cases:

- Case 1: What is the uncertainty of $\mathrm{CO}_{2}$ capture fraction due to the uncertainties in the input parameters (i.e., those involving equilibrium constants and mass transfer)?

- Case 2: What is the uncertainty of lean solvent flow rate due to the same input uncertainties assuming $\mathrm{CO}_{2}$ capture fraction is constrained to be approximately $90 \%$ ?

- Case 3: If we consider lean loading as an additional uncertain input, what is the uncertainty of the lean solvent flow rate due to the input uncertainties assuming $\mathrm{CO}_{2}$ capture fraction is constrained to be approximately $90 \%$ ?

These cases were suggested by the SMEs as useful scenarios for understanding how the MEA system behaves under uncertainty of the inputs.

\subsection{MODEL V1}

The uncertain inputs for Model V1, along with their uncertainty intervals, are summarized in Table 5-1. Each input is attributed a uniform distribution as described in Section 4.1.

Table 5-1: Input parameters for Model V1

\begin{tabular}{|l|l|l|l|l|l|}
\hline Inputs & Min & Max & Case 1 & Case 2 & Case 3 \\
\hline $\mathrm{A}_{1}$ & 0.6942 & 0.8949 & $\mathrm{X}$ & $\mathrm{X}$ & $\mathrm{X}$ \\
\hline $\mathrm{A}_{2}$ & 98.4606 & 98.6613 & $\mathrm{X}$ & $\mathrm{X}$ & $\mathrm{X}$ \\
\hline $\mathrm{A}_{3}$ & 215.9436 & 216.1442 & $\mathrm{X}$ & $\mathrm{X}$ & $\mathrm{X}$ \\
\hline $\mathrm{A}_{4}$ & 1.1772 & 1.3779 & $\mathrm{X}$ & $\mathrm{X}$ & $\mathrm{X}$ \\
\hline $\mathrm{A}_{5}$ & 132.7936 & 132.9943 & $\mathrm{X}$ & $\mathrm{X}$ & $\mathrm{X}$ \\
\hline $\mathrm{C}_{\mathrm{L}}$ & 1.9694 & 2.6306 & $\mathrm{X}$ & $\mathrm{X}$ & $\mathrm{X}$ \\
\hline $\mathrm{C}_{\mathrm{V}}$ & 0.1727 & 0.2673 & $\mathrm{X}$ & $\mathrm{X}$ & $\mathrm{X}$ \\
\hline Lean Loading & 0.1600 & 0.3500 & & & $\mathrm{X}$ \\
\hline
\end{tabular}

For each case, two sample sets were generated, one using the LPTAU (which is a quasi-Monte Carlo) sampling scheme and the other using the Morris sampling scheme. (The Morris sample is used for parameter screening.) Table 5-2 summarizes the sample size for each case, where "M/N" means that only $\mathrm{M}$ samples were successful out of the $\mathrm{N}$ samples that were provided to the Aspen Plus server. Thus, the subsequent UQ analyses were performed on the M samples. 
Table 5-2: Sample size of Model V1 simulation data

\begin{tabular}{|l|l|l|l|}
\hline \multicolumn{1}{|c|}{ Sample scheme } & Case 1 & Case 2 & Case 3 \\
\hline LPTAU & $989 / 1000$ & $998 / 1000$ & $754 / 1000$ \\
\hline MOAT & $160 / 160$ & $160 / 160$ & $106 / 180$ \\
\hline
\end{tabular}

\section{Case 1}

With the specified input ranges (cf. Table 5-1), we found that the output, which is $\mathrm{CO}_{2}$ capture fraction, follows the probability distribution (and corresponding cumulative density function) shown in Figure 5-1.
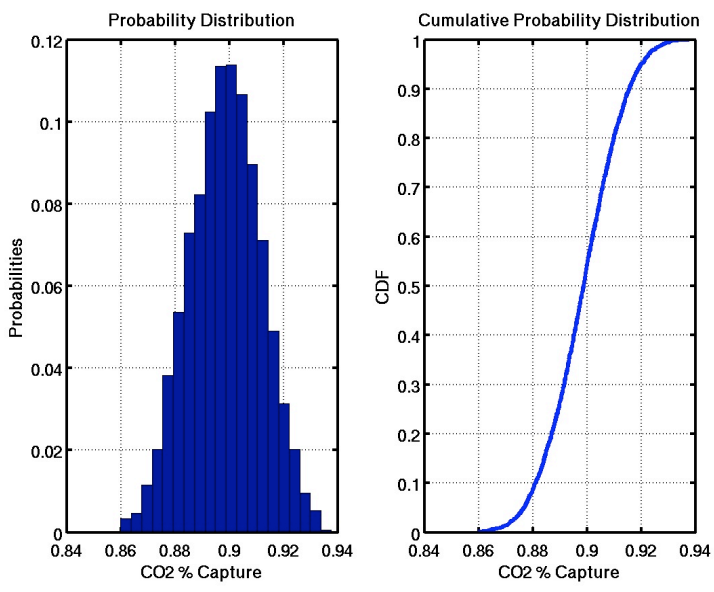

Figure 5-1: Output PDF and CDF from forward uncertainty analysis for Model V1, Case 1

Next, we tried to fit a response surface to approximate the input-to-output mapping from Model V1's simulation data. The UQ Toolset provides a suite of response surface models, along with validation tools for one to examine the goodness-of-fit. Of the multiple response surface models, splines appear to provide an adequate fit for the input-output mapping. An adequate fit is signified by an error histogram with values close to 0 (left) and a near-diagonal line in the actual vs. predicted data plot (right), as shown in Figure 5-2. 

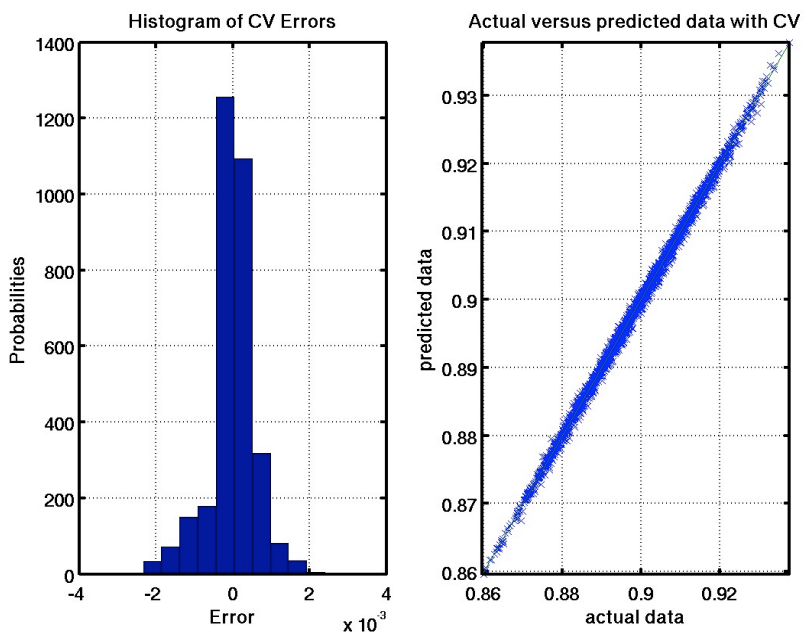

Figure 5-2: Validation plots from response surface analysis for Model V1, Case 1

Next, we used the validated response surface to perform variance-based sensitivity analysis to identify the most important parameters. We found that the three most sensitive input parameters are $A_{1}, A_{2}$ and $A_{3}$. We used the response surface visualization capability to plot the response surface that approximates the mapping from $\left(\mathrm{A}_{1}, \mathrm{~A}_{2}, \mathrm{~A}_{3}\right)$ to the $\mathrm{CO}_{2}$ capture fraction. Figure 5-3 shows the 3-D response surface that displays the $\left(\mathrm{A}_{1}, \mathrm{~A}_{2}, \mathrm{~A}_{3}\right)$ space (the colored region) that would achieve approximately $90 \% \mathrm{CO}_{2}$ capture fraction.

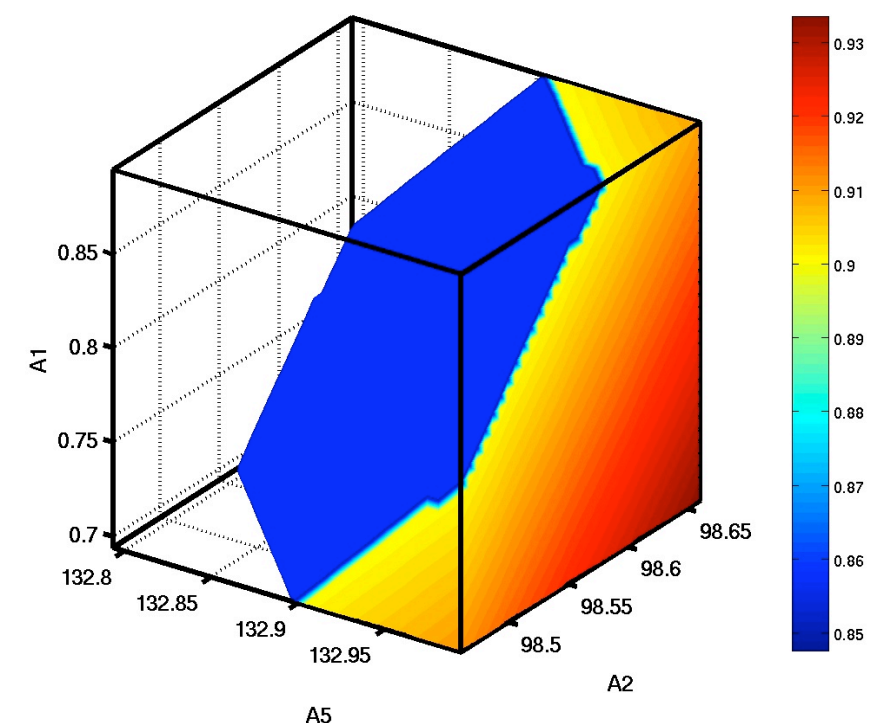

Figure 5-3: 3-D response surface visualization for Model V1, Case 1 


\section{Case 2}

For Case 2, Model V1 was run with the user-specified constraint of approximately $90 \% \mathrm{CO}_{2}$ capture fraction. To satisfy this constraint, the Aspen Plus environment internally optimized the model parameters. Given the same input uncertainties specified in Table 5-1, the lean solvent flow rate follows the probability distribution shown in Figure 5-4.

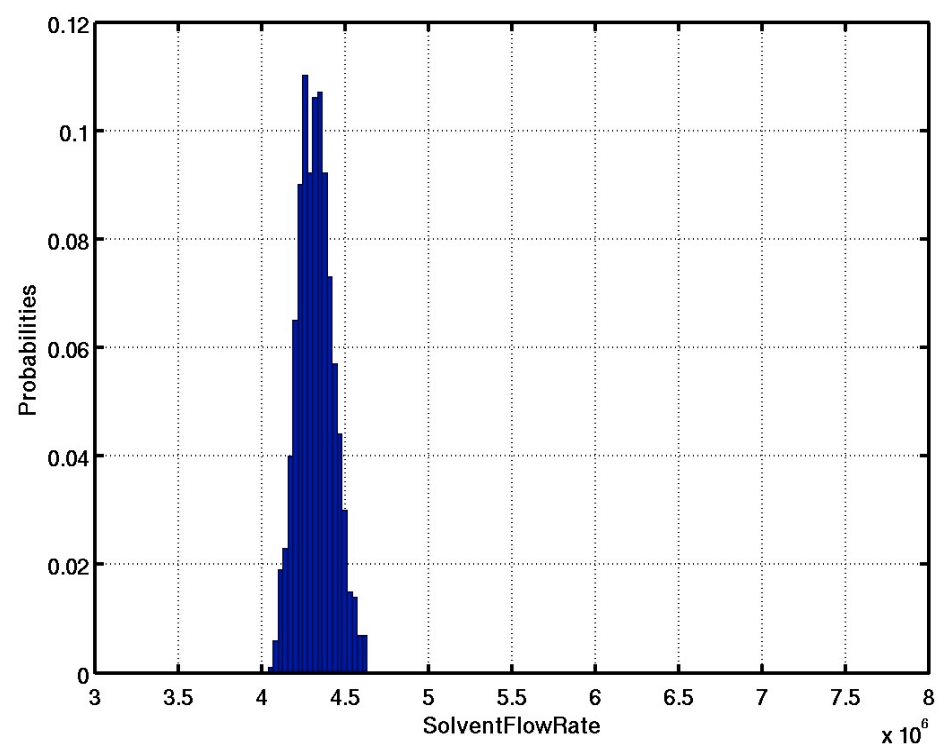

Figure 5-4: Output PDF from forward uncertainty analysis for Model V1, Case 2

Applying variance-based sensitivity analysis, the impact of each input on the output (i.e., lean solvent flow rate) is displayed as a pie chart in Figure 5-5. Here, the most sensitive inputs are identified to be $A_{1}$, $\mathrm{A}_{2}$ and $\mathrm{A}_{5}$. The pie chart shows how much each input parameter contributes to the output variance.

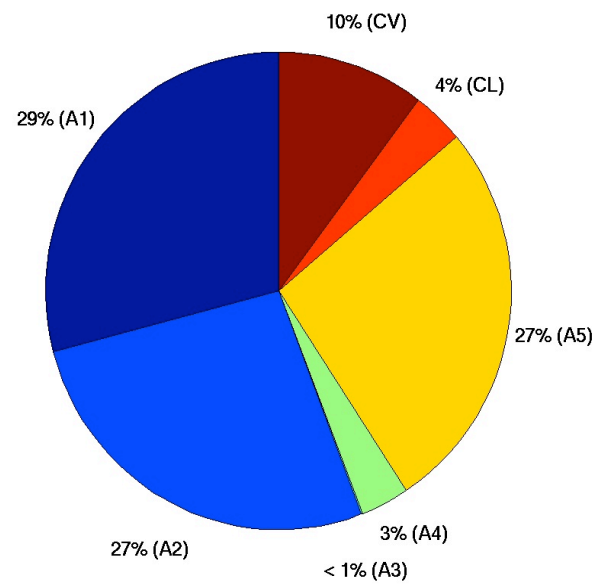

Figure 5-5: Pie chart from sensitivity analysis for Model V1, Case 2 
Parameter sensitivities can also be assessed visually as scatter plots, as in Figure 5-6. A sensitive input would exhibit a clear upward or downward trend in its scatter plot. Here, we observe such a trend for $A_{1}$, $\mathrm{A}_{2}$ and $\mathrm{A}_{5}$.
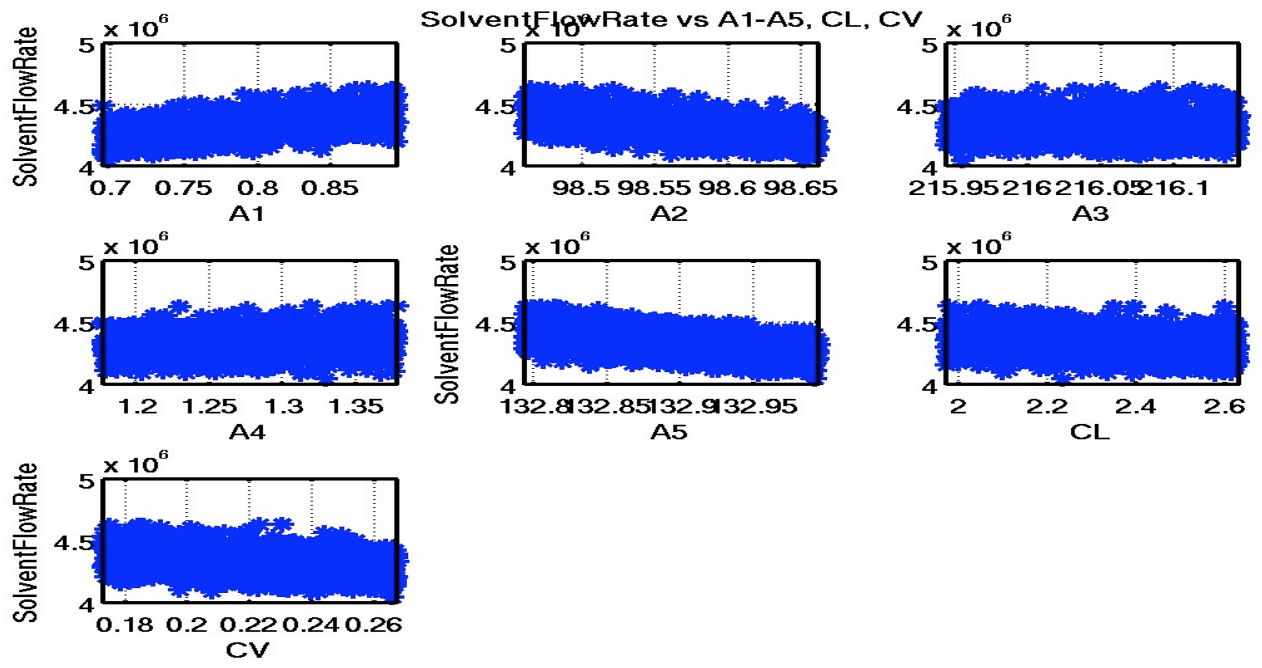

Figure 5-6: Scatter plots from sensitivity analysis for Model V1, Case 2

\section{Case 3}

For Case 3, the process model was run with the same settings as in Case 2, with the addition of lean loading as an extra input parameter. Given the input uncertainties specified in Table 5-1, the lean solvent flow rate follows the probability distribution shown in Figure 5-7.

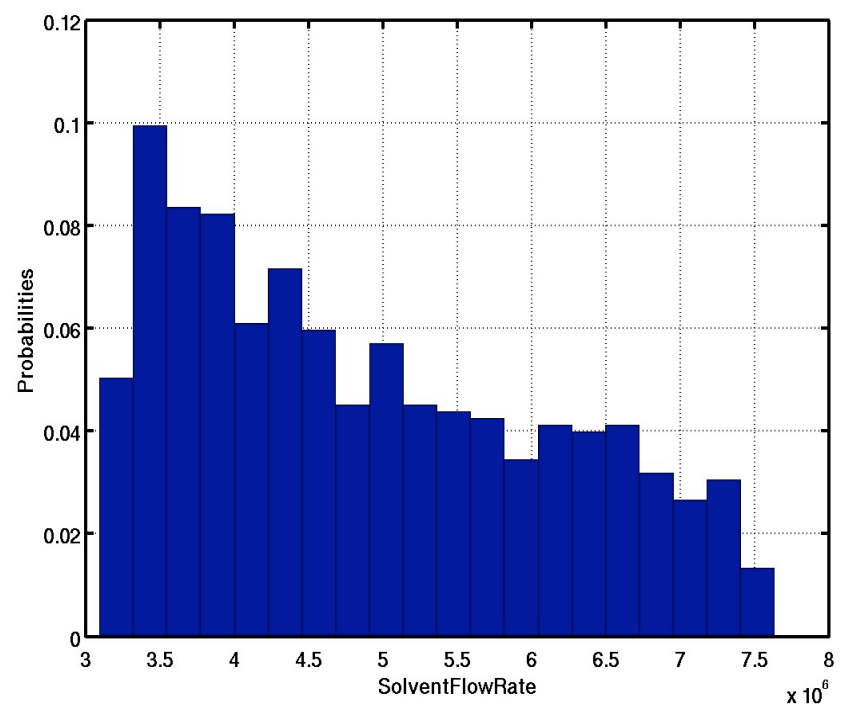

Figure 5-7: Output PDF from forward uncertainty analysis for Model V1, Case 3 
Comparing Figure 5-7 to Figure 5-4, one can see that the inclusion of lean loading as an extra uncertain input had drastically made the output (i.e., lean solvent flow rate) more uncertain. This is confirmed by parameter screening, also known as variable selection, in which the lean solvent flow rate (shown as Variable 8 in Figure 5-7) is ranked the highest compared to the other inputs. This figure is generated using the Morris parameter screening analysis.

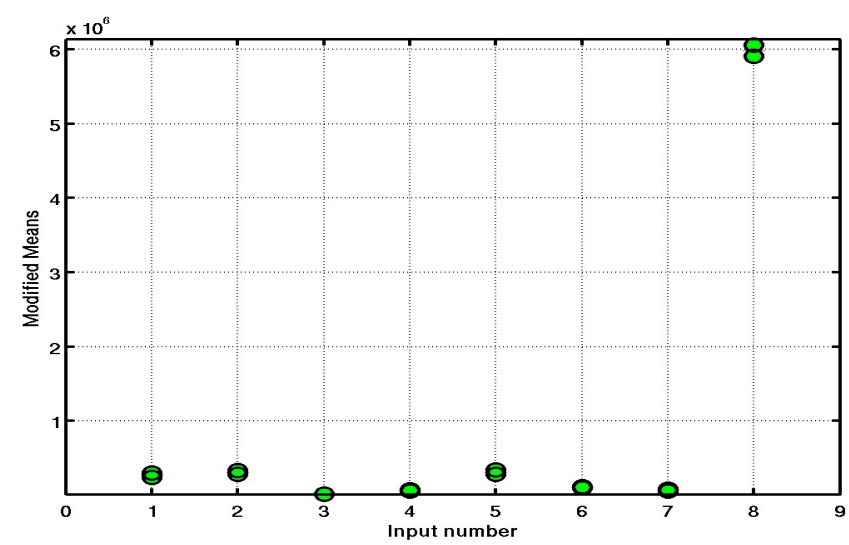

Figure 5-8: Bootstrap modified means plot from parameter screening for Model V1, Case 3

Lastly, we assumed that the lean solvent flow rate was observed and its measured value is $4.3244 \mathrm{e} 6$ with standard deviation of $1 \mathrm{e} 5$. (This is a manufactured observation datum to demonstrate Bayesian inference.) We performed inverse uncertainty analysis to infer the input uncertainties that could have resulted in such an observation. The PDFs (also referred to as posterior distributions) of the uncertain inputs are shown in Figure 5-9. Note that before the observation, the lean loading was assumed to be within the range [0.16, 0.35]. After the observation, the lean loading is now shown to be within a much narrower range, which shows that the observation of lean solvent flow rate drastically reduced the uncertainty about the lean loading. This agrees with the other UQ analyses that confirm that there is tight coupling between the lean loading and the lean solvent flow rate.
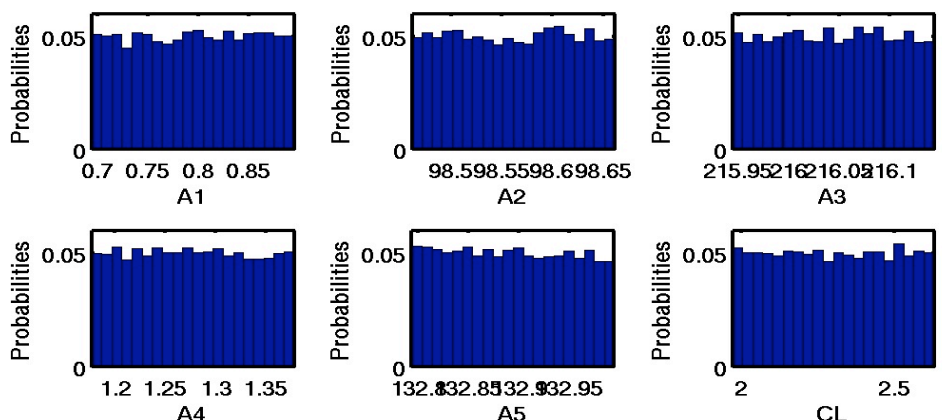

A5
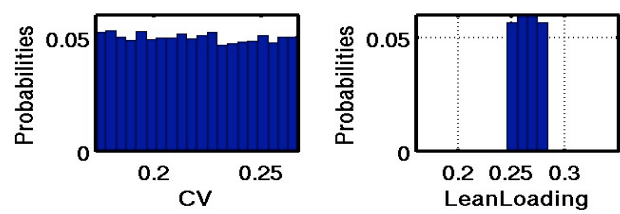

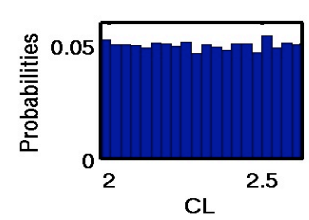

CL

Figure 5-9: Input posteriors from Bayesian inference for Model V1, Case 3 


\subsection{MODEL V2}

The uncertain inputs for Model V2, along with their uncertainty intervals, are summarized in Table 5-3. Each input is attributed a uniform distribution as described in Section 4.2.

Table 5-3: Input parameters for Model V2

\begin{tabular}{|l|l|l|l|l|l|}
\hline Inputs & Min & Max & Case 1 & Case 2 & Case 3 \\
\hline$\alpha_{4}$ & $3.8448 \mathrm{e} 13$ & $4.7952 \mathrm{e} 13$ & $\mathrm{X}$ & $\mathrm{X}$ & $\mathrm{X}$ \\
\hline$\alpha_{5}$ & $2.1182 \mathrm{e} 17$ & $2.6418 \mathrm{e} 17$ & $\mathrm{X}$ & $\mathrm{X}$ & $\mathrm{X}$ \\
\hline$\alpha_{6}$ & $9.0861 \mathrm{e} 10$ & $1.0453 \mathrm{e} 11$ & $\mathrm{X}$ & $\mathrm{X}$ & $\mathrm{X}$ \\
\hline$\alpha_{7}$ & $3.2399 \mathrm{e} 19$ & $3.2300 \mathrm{e} 19$ & $\mathrm{X}$ & $\mathrm{X}$ & $\mathrm{X}$ \\
\hline$\Delta \mathrm{T}{ }_{1}$ & $-3.2921 \mathrm{e}-01$ & $2.9904 \mathrm{e}-01$ & $\mathrm{X}$ & $\mathrm{X}$ & $\mathrm{X}$ \\
\hline$\Delta \mathrm{T}_{2}$ & $-4.7760 \mathrm{e}-01$ & $4.3465 \mathrm{e}-01$ & $\mathrm{X}$ & $\mathrm{X}$ & $\mathrm{X}$ \\
\hline$\Delta \mathrm{T}_{3}$ & $\begin{array}{l}\text { Not enough info; currently omitted } \\
\text { from study }\end{array}$ & & & & \\
\hline $\mathrm{a}_{\mathrm{e}}$ & $9.000 \mathrm{e}-01$ & $1.100 \mathrm{e} 00$ & $\mathrm{X}$ & $\mathrm{X}$ & $\mathrm{X}$ \\
\hline Lean Loading & $2.200 \mathrm{e}-01$ & $3.000 \mathrm{e}-01$ & & & $\mathrm{X}$ \\
\hline
\end{tabular}

For each case, one LPTAU sample set was generated. Table 5-4 summarizes the sample size for each case, where "M/N" means that only $\mathrm{M}$ samples were successful out of the $\mathrm{N}$ samples that were provided to the Aspen Plus server. Thus, the subsequent UQ analyses were performed on the M samples.

Table 5-4: Sample size of Model V2 simulation data

\begin{tabular}{|l|l|l|l|}
\hline Sample scheme & Case 1 & Case 2 & Case 3 \\
\hline LPTAU & $9992 / 10000$ & $10000 / 10000$ & $9375 / 10000$ \\
\hline
\end{tabular}


We had chosen to use such a large sample size in order to help test the robustness of the interface developed by the Integration Framework Team. Such a large sample size is not necessary and the same UQ analyses could be performed on a smaller sample.

\section{Case 1}

With the prescribed input uncertainty ranges (cf. Table 5-3), we observe that the output, which is $\mathrm{CO}_{2}$ capture fraction, follows the probability distribution (and corresponding cumulative density function) shown in Figure 5-10.
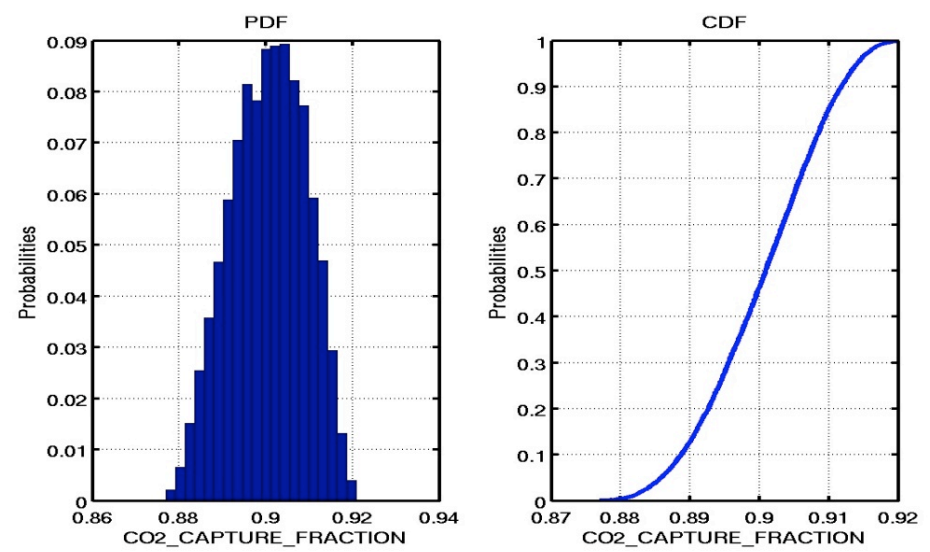

Figure 5-10: Output PDF and CDF from forward uncertainty analysis for Model V2, Case 1

Next, we performed a response surface analysis to approximate the input-to-output mapping from Model V2's simulation data for Case 1. Figure 5-11 shows a comparison between a linear response model (right) with a quadratic response model. Here, a diagonal line on the right plot means that the response model interpolates well and is an adequate approximation for the input-to-output mapping. In addition, one can also examine the coefficient of determination $\mathrm{R}^{2}$, which represents the proportion of variability in a data set that is accounted for by the fitted model. Here, the closer $\mathrm{R}^{2}$ is to 1 , the better the regression fits the data. In Figure 5-11, the left plot shows the linear response model with $\mathrm{R}^{2}=0.997$, and the right plot shows the (superior) quadratic response model with $\mathrm{R}^{2}$ close to 1 .
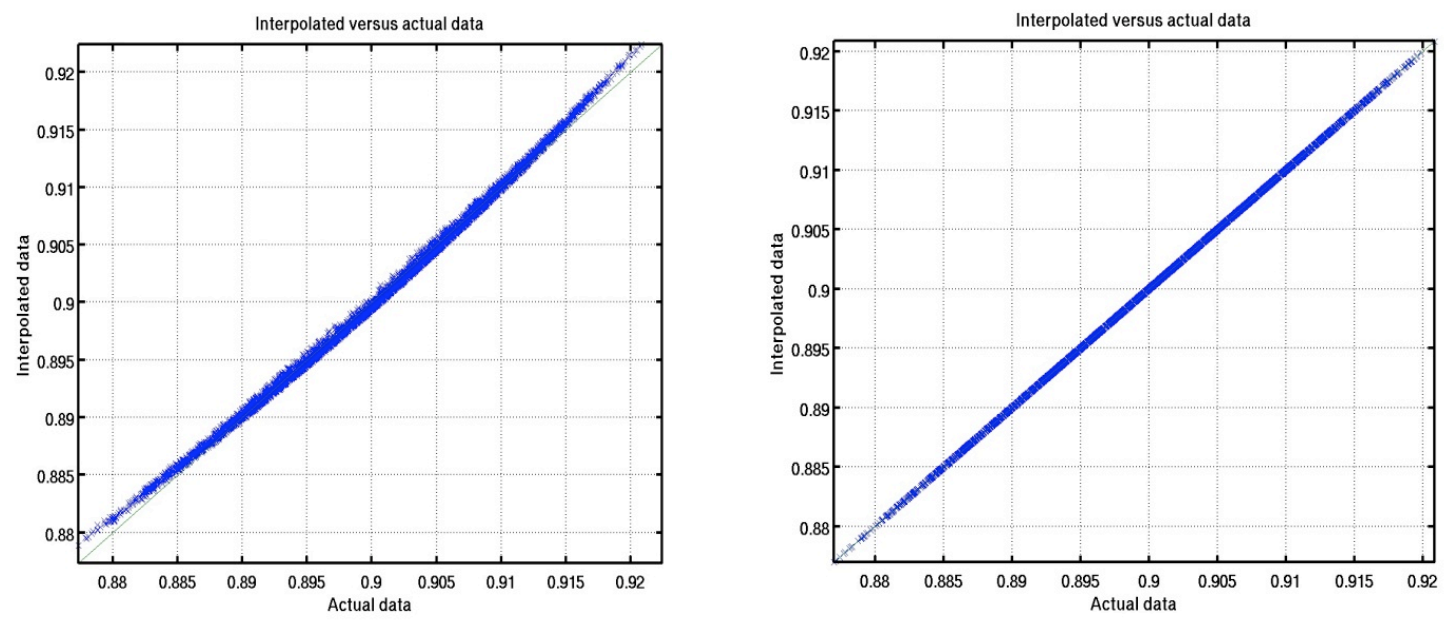

Figure 5-11: Validation plots from response surface analysis for Model V2, Case 1 
We the used the validated response surface to perform variance-based sensitivity analysis and identified $\alpha_{6}$ and $\mathrm{a}_{\mathrm{e}}$ as the two most important inputs, with $\alpha_{6}$ contributing to $23.4 \%$ and $\mathrm{a}_{\mathrm{e}}$ contributing to $72.7 \%$ to the overall uncertainty of the $\mathrm{CO}_{2}$ capture fraction. As before, the scatter plots with clear upward or downward trends correspond to the inputs that are most sensitive. Figure 5-12 (which labels $\alpha_{6}$ as "A6" and $\mathrm{a}_{\mathrm{e}}$ as "AE") confirms that $\alpha_{6}$ and $\mathrm{a}_{\mathrm{e}}$ are the two most sensitive inputs.
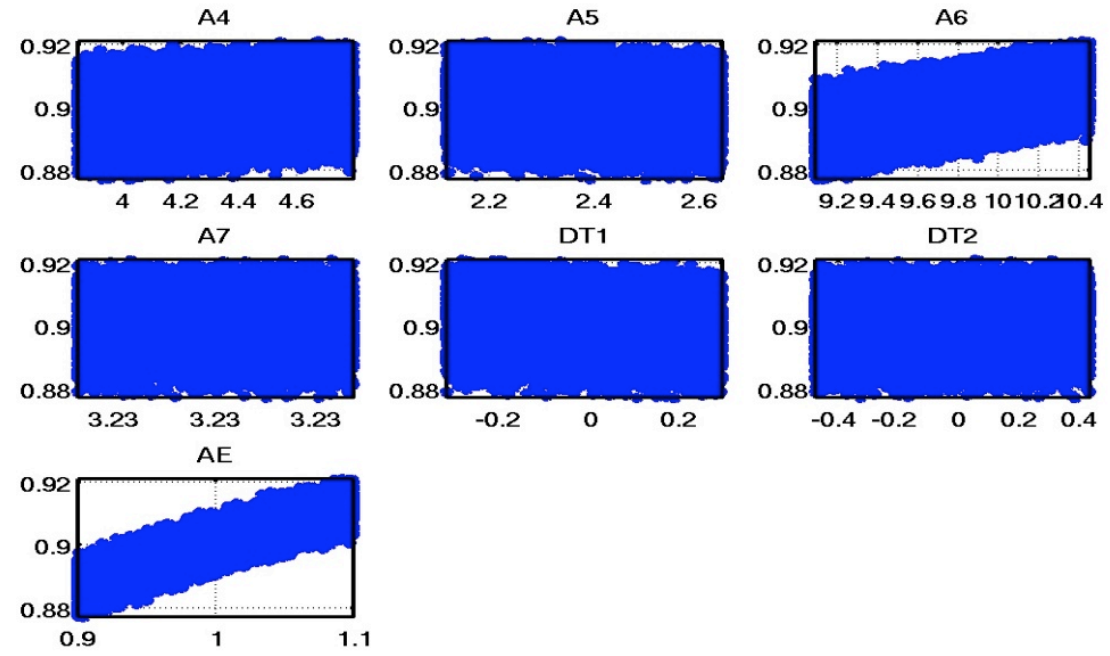

Figure 5-12: Scatter plots from sensitivity analysis analysis for Model V2, Case 1

Once we have identified the important input parameters, we can use response surface visualization to plot the response surface that approximates the mapping from the important inputs $\left(\alpha_{6}\right.$ and $\left.a_{e}\right)$ to the output $\left(\mathrm{CO}_{2}\right.$ capture fraction). Figure 5-13 shows the 2-D response surface that displays the $\left(\alpha_{6}, \mathrm{a}_{\mathrm{e}}\right)$ space (colored region) that would achieve approximately $90 \% \mathrm{CO}_{2}$ capture fraction.

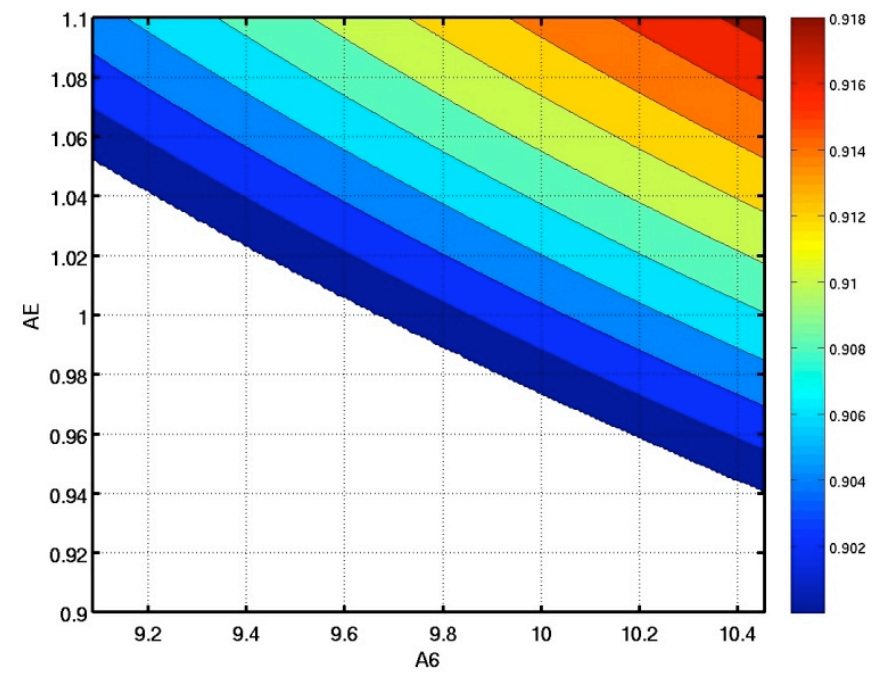

Figure 5-13: 2-D response surface visualization for Model V2, Case 1 


\section{Case 2}

For Case 2, Model V2 was run with the user-specified constraint of approximately $90 \% \mathrm{CO}_{2}$ capture fraction. To satisfy this constraint, the Aspen Plus environment internally optimized the model parameters. Given the same input uncertainties specified in Table 5-3, the lean solvent flow rate was observed to follow the probability distribution shown in Figure 5-14.
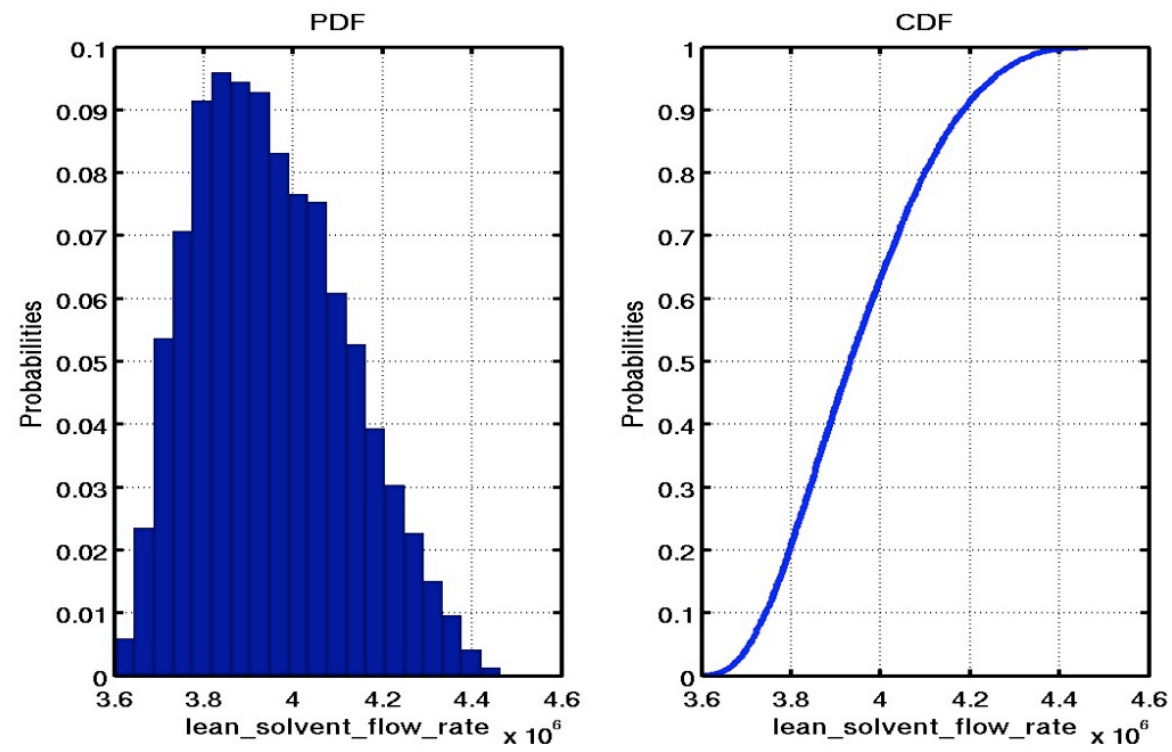

Figure 5-14: Output PDF and CDF from forward uncertainty analysis for Model V2, Case 2

Next, we performed a response surface analysis to approximate the input-to-output mapping from Model V2's simulation data for Case 2. Figure 5-15 shows a comparison between a linear response model (left) with a quadratic response model (right). Linear regression gives $\mathrm{R}^{2}=0.985$, while quadratic regression gives a more superior $\mathrm{R}^{2}=0.999$.
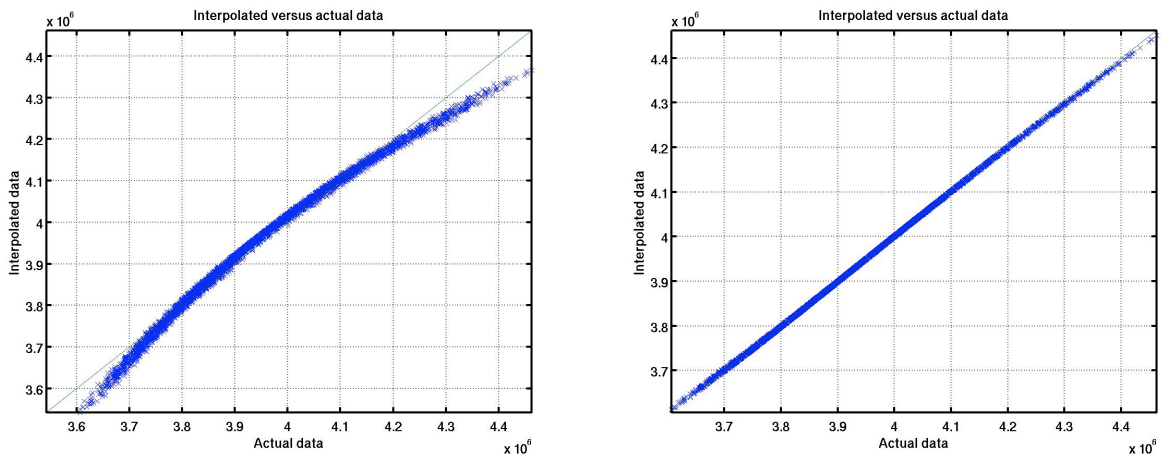

Figure 5-15: Validation plots from response surface analysis for Model V2, Case 2

Using variance-based sensitivity analysis, we identified $\alpha_{6}$ and $a_{e}$ as the two most important inputs, with $\alpha_{6}$ contributing to $22.9 \%$ and $\mathrm{a}_{\mathrm{e}}$ contributing to $73.1 \%$ to the overall uncertainty of the lean solvent flow rate. As before, the scatter plots with clear upward or downward trends correspond to the inputs that are 
most sensitive. Figure 5-16 (which labels $\alpha_{6}$ as "A6" and $a_{e}$ as "AE") confirms that $\alpha_{6}$ and $a_{e}$ are the two most sensitive inputs.
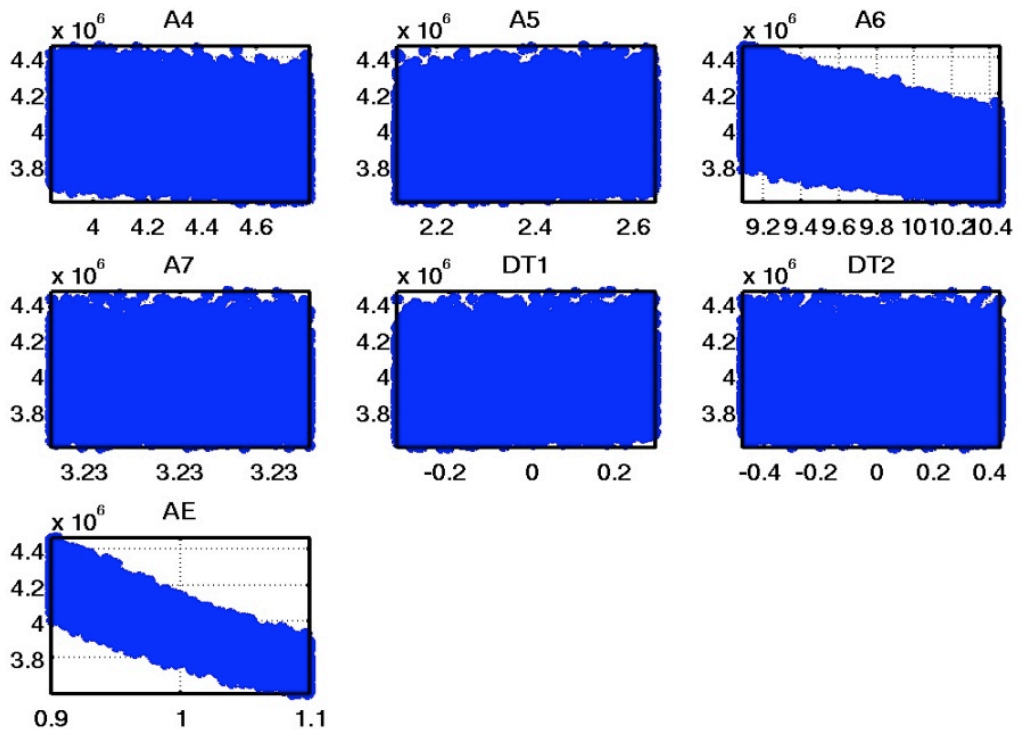

Figure 5-16: Scatter plots from sensitivity analysis analysis for Model V2, Case 2

Once we have identified the important input parameters, we can use response surface visualization to plot the response surface that approximates the mapping from the important inputs $\left(\alpha_{6}\right.$ and $\left.a_{e}\right)$ to the output (lean solvent flow rate), as shown in Figure 5-17.
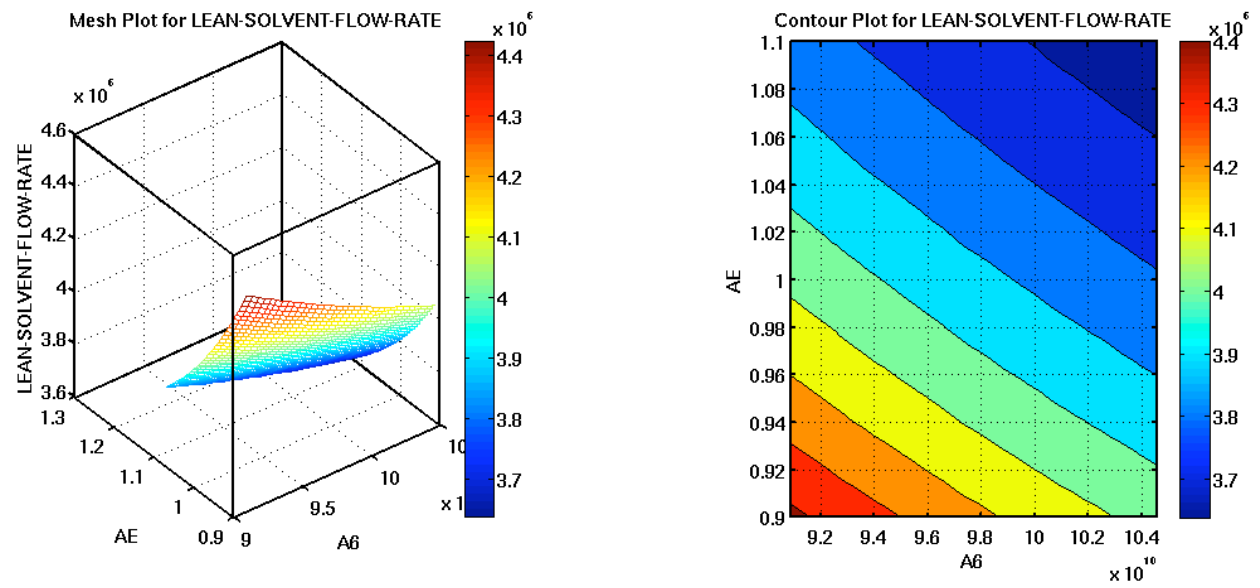

Figure 5-17: 2-D response surface visualization for Model V2, Case 2

\section{Case 3}

For Case 3, the process model was run with the same settings as in Case 2, with the addition of lean loading as an extra input parameter. Given the input uncertainties specified in Table 5-3, the lean solvent flow rate was observed to follow the probability distribution shown in Figure 5-18. Comparing Figure 518 to Figure 5-14, one can see that the inclusion of lean loading as an extra uncertain input had drastically made the output (i.e., lean solvent flow rate) more uncertain. 


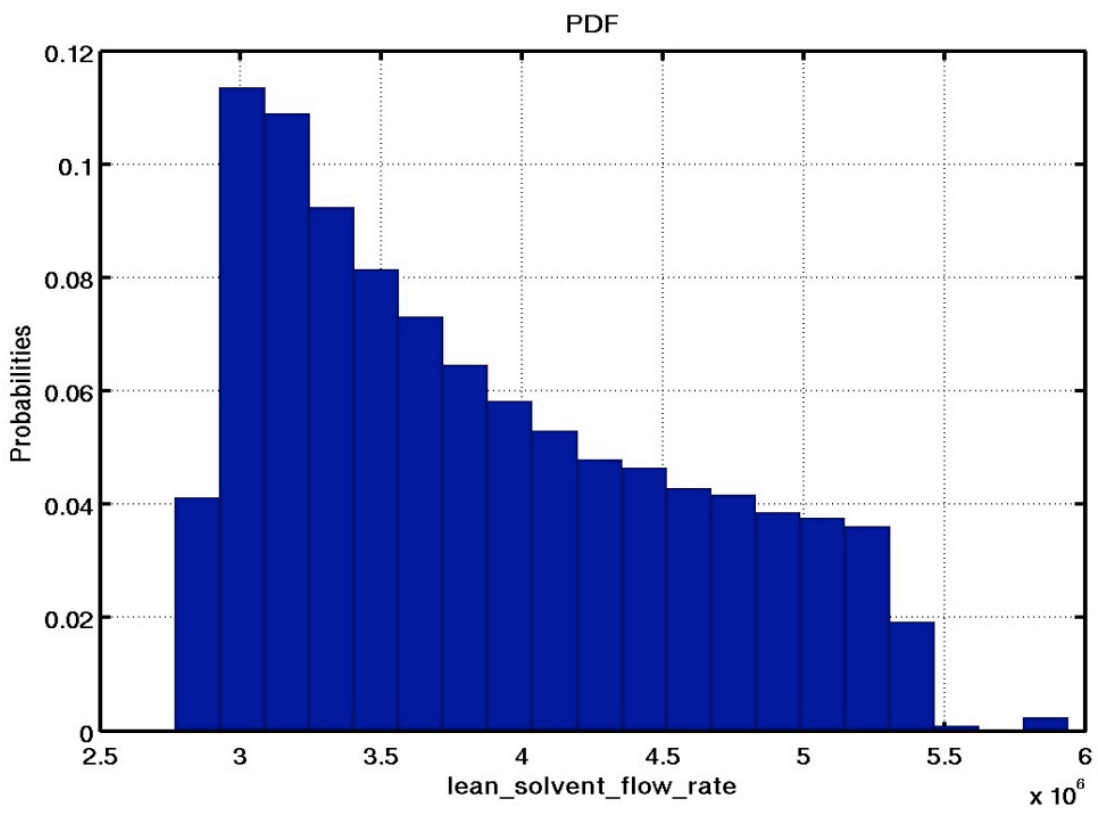

Figure 5-18: Output PDF from forward uncertainty analysis for Model V2, Case 3

Again, we performed a response surface analysis to approximate the input-to-output mapping from Model V2's simulation data for Case 3. Figure 5-19 shows a comparison between a cubic response model (right) with a multivariate splines response model. Here, a diagonal line means that the response model interpolates well and is an adequate approximation for the input-to-output mapping. Both response models' performance degrades drastically when the lean solvent flow rate is greater than 5.5. The reason for this degradation is due to the lack of data for this range of lean solvent flow rate, which might be attributed to the fact that, under the constraint of approximately $90 \% \mathrm{CO}_{2}$ capture fraction, it is difficult to obtain valid simulation runs given the specified input uncertainties.
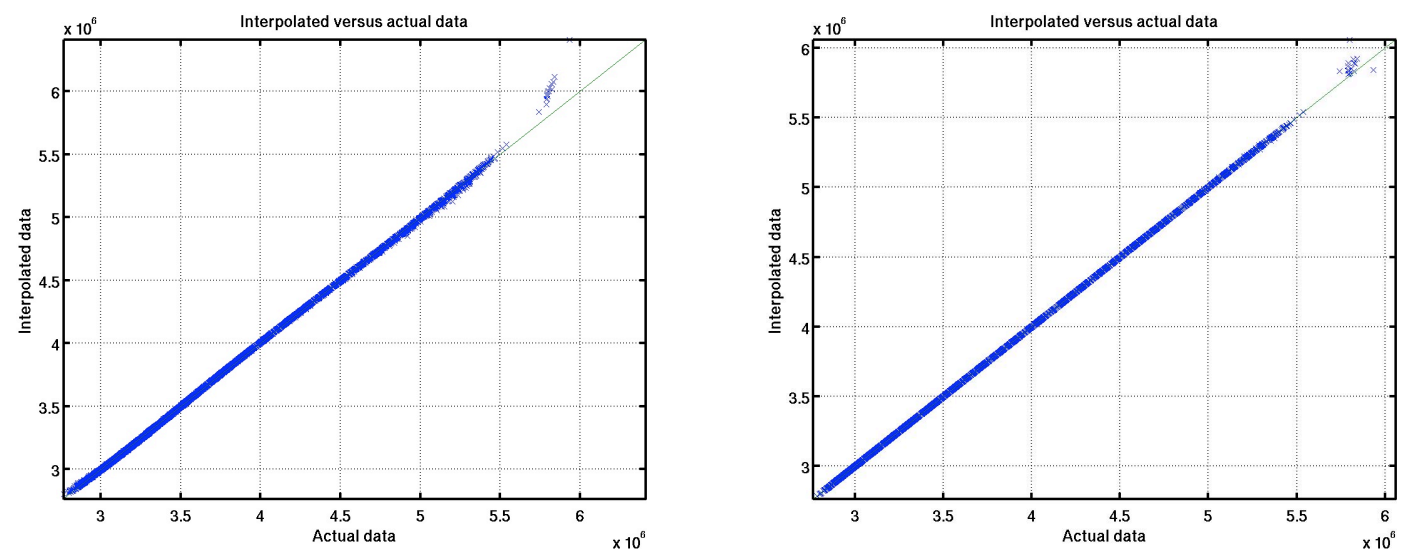

Figure 5-19: Validation plots from response surface analysis for Model V2, Case 3

Lastly, we performed variance-based sensitivity analysis and lean loading as the most important input, contributing to $95.7 \%$ to the overall uncertainty of the lean solvent flow rate. As before, the scatter plots with clear upward or downward trends correspond to the inputs that are most sensitive. Figure 5-20 
confirms that lean loading is the most sensitive input. Moreover, it also explains why the inclusion of lean loading as an extra input led to such drastic changes in the output PDF.
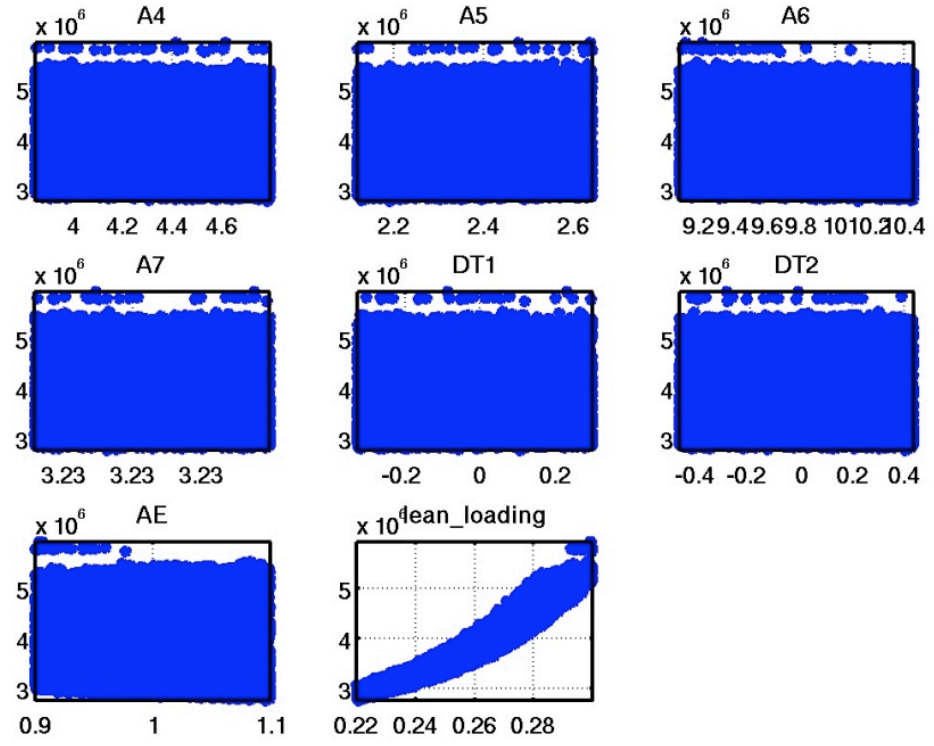

Figure 5-20: Scatter plots from sensitivity analysis analysis for Model V2, Case 3

Once we have identified the important input parameters, we can use response surface visualization to plot the response surface that approximates the mapping from lean loading to the lean solvent flow rate, as shown in Figure 5-21.

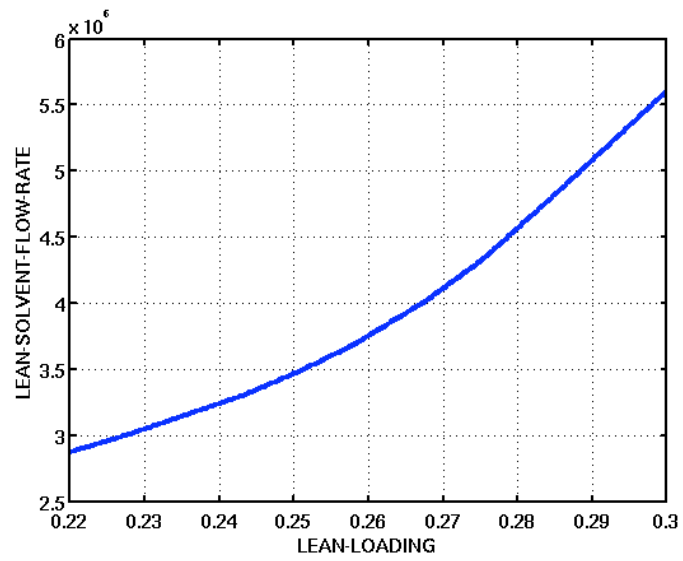

Figure 5-21: 1-D response surface visualization for Model V2, Case 3 


\subsection{Summary \& Future Work}

In Year 1 of the current CCSI project, we have made good progress in understanding the uncertainties associated with some carbon capture models (in this case, MEA) as well as in developing an initial set of capabilities for UQ analysis, which comprises several UQ techniques such as parameter screening, response surface analysis, quantitative sensitivity analysis and parameter estimation. In addition, partnering with the Integration Framework Team, we have designed and implemented a graphical user interface suitable for performing UQ on CCSI models. Our UQ Toolset, along with its graphical user interface, has been demonstrated on two MEA models as described in Sections 4 and 5.

Future work consists of extending the UQ Toolset to include additional capabilities to deal with more complex carbon capture process and CFD models, and demonstrating the toolset on these more complex models. We will also address the challenge of performing on scaled-up systems where no experimental data are available. Additionally, in collaboration with the Integration Framework Team, work is underway to improve the userability of the graphical user interface. We are currently eliciting feedback from user studies and enhancing the presentation of the UQ workflow to the user to make the analysis process more intuitive and seamless. 


\subsection{References}

D. M. Austgen, G. T. Rochelle, X. Peng, C. Chen, "Model of Vapor-Liquid Equilibria for Aqueous Acid Gas-Alkanolamine Systems Using the Electrolyte-NRTL Equation," Ind. Eng. Chem. Res., 1989, 28(7), 1060-1073.

R. Billet, M. Schultes, "Predicting Mass Transfer in Packed Columns," Chem. Eng. Tech., 1993, 16, 1-9.

B. Hanley, C. Chen, "New Mass-Transfer Correlations for Packed Towers", AIChE J., 2012, 58(1), 132152.

H. Hikita, S. Asai, H. Ishikawa, M. Honda, "The Kinetics of Reactions of Carbon Dioxide with Monoethanolamine, Diethanolamine, and Triethanolamine by a Rapid Mixing Method," Chem. Eng. J., 1977, 13, 7-12.

B.R. Pinsent, L. Pearson, F.J.W. Roughton, "The Kinetics of Combination of Carbon Dioxide with Hydroxide Ions," Trans. Faraday Soc., 1956, 52, 1512-1520.

C.H. Tong, "PSUADE User's Manual, Version 1.2.0,” Lawrence Livermore National Laboratory, Livermore, CA, Software Manual LLNL-SM-407882, May 2009.

G.Q. Wang, X.G. Yuan, K.T. Yu, "Review of Mass-Transfer Correlations for Packed Columns," Ind. Eng. Chem. Res., 2005, 44(23), 8715-8729. 


\subsection{Glossary}

\begin{tabular}{|l|l|}
\hline Acronym & Descriptive Name \\
\hline ARRA & American Recovery and Reinvestment Act of 2009 \\
\hline CCSI & Carbon Capture Simulation Initiative \\
\hline CDF & Cumulative Distribution Function \\
\hline CO $_{2}$ & Carbon Dioxide \\
\hline D & Dimension (For example: 3-D visualization) \\
\hline FWP & Field Work Proposal \\
\hline GUI & Graphical User Interface \\
\hline HETP & Height Equivalent to a Theoretical Plate \\
\hline ICP & Industrially-relevant Challenge Problem(s) \\
\hline LANL & Los Alamos National Laboratory \\
\hline LBNL & Lawrence Berkeley National Laboratory \\
\hline LH & Latin Hypercube \\
\hline LLNL & Lawrence Livermore National Laboratory \\
\hline MEA & Monoethanolamine \\
\hline MC & Monte Carlo \\
\hline MCMC & Markov Chain Monte Carlo \\
\hline NETL & National Energy Technology Laboratory \\
\hline OA & Orthogonal Array \\
\hline PC & Pulverized Coal \\
\hline PDF & Probability Density Function \\
\hline PNNL & Pacific Northwest National Laboratory \\
\hline PSUADE & Problem Solving Environment for Uncertainty Analysis and Design Exploration \\
\hline QMC & Quasi-Monte Carlo \\
\hline UQ & Uncertainty Quantification \\
\hline V & Version (For example: V1 refers to Version 1) \\
\hline & \\
\hline
\end{tabular}

